Homology, Homotopy and Applications, vol.19(2), 2017, pp.373-395

\title{
TATE OBJECTS IN STABLE $(\infty, 1)$-CATEGORIES
}

\author{
BENJAMIN HENNION
}

(communicated by J.P.C. Greenlees)

\begin{abstract}
Tate objects allow us to deal with infinite dimensional spaces by identifying some more structure. In this article, we set up the theory of Tate objects in stable $(\infty, 1)$-categories, while the literature only deals with exact categories. We will prove the main properties expected from Tate objects. In particular, we show that the K-theory of Tate objects is a delooping of that of the original category. This gives us a procedure to transport invariants from finite dimensional objects to Tate objects, hence providing interesting invariants.

This new setting includes several useful examples: Tate objects in the category of spectra for instance, or in the derived category of a derived algebraic object - which can be thought of as a structured infinite dimensional vector bundle in the derived setting.
\end{abstract}

\section{Introduction}

Tate vector spaces have been used by many to deal with infinite dimensional spaces. Identifying some structure allows us to define a well-behaved duality on infinite dimensional spaces. They were studied by several authors, including Lefschetz, Beilinson, Drinfeld and more recently Osipov and Zhu, Previdi, Saito, and Bräunling, Gröchenig and Wolfson.

In [Dri], Drinfeld describes them the following way. Let us fix a field $k$. Let $V$ be a vector space, which we see as a discrete topological space. Its topological dual $V^{\vee}$ is then what is called a linearly compact vector space. A Tate vector space is a topological space of the form $V \oplus W^{\vee}$ where both $V$ and $W$ are discrete topological vector spaces. The first example of such a Tate vector space is the field of Laurent series $k((t)) \simeq k \llbracket t \rrbracket \oplus t^{-1} k\left[t^{-1}\right]$. For any Tate vector space $X$, we then have $\left(X^{\vee}\right)^{\vee} \simeq X$.

Bräunling, Gröchenig and Wolfson $[\mathrm{BGW}]$ generalised the idea of Tate vector spaces to Tate objects in an exact category $\mathcal{C}$ - objects of $\mathcal{C}$ playing the role of finite dimensional vector spaces. They use a categorical version of discrete infinite dimensional objects (namely $\operatorname{Ind}(\mathcal{C})$ ) and of linearly compact objects (namely $\operatorname{Pro}(\mathcal{C})$ ). An elementary Tate object is then an object $X$ that fits in an exact sequence

$$
X^{p} \rightarrow X \rightarrow X^{i}
$$

Received August 5, 2016, revised January 26, 2017, April 4, 2017; published on November 29, 2017. 2010 Mathematics Subject Classification: 18F25, 18 G55.

Key words and phrases: Tate object, higher category, K-theory.

Article available at http://dx.doi.org/10.4310/HHA.2017.v19.n2.a18

Copyright (C) 2017, Benjamin Hennion. Permission to copy for private use granted. 
where $X^{i}$ is discrete and $X^{p}$ is linearly compact. A Tate object is a retract of an elementary Tate object. For instance the field $\mathbb{Q}_{p}$ is a Tate object in abelian groups, as it fits in the sequence

$$
\mathbb{Z}_{p} \rightarrow \mathbb{Q}_{p} \rightarrow \mathbb{Q}_{p} / \mathbb{Z}_{p}
$$

In this paper, we establish a suitable framework to talk about derived categories of Tate modules. Examples of such "Tate complexes" can be found in formal algebraic geometry (the cohomology of a punctured formal neighbourhood is a Tate complex, for instance). As recent developments have shown, a suitable framework to talk about categories of complexes is that of stable $\infty$-categories. Those are the higher categorical version of triangulated categories. We therefore define Tate objects in a stable $\infty$ category $\mathcal{C}$ by first identifying a category $\operatorname{Ind}(\mathcal{C})$ of discrete objects and a category $\operatorname{Pro}(\mathcal{C})$ of linearly compact objects. The category $\operatorname{Tate}(\mathcal{C})$ of Tate objects in $\mathcal{C}$ is then composed of retracts of extensions $X^{p} \rightarrow X \rightarrow X^{i}$ as above, with $X^{p} \in \operatorname{Pro}(\mathcal{C})$ and $X^{i} \in \operatorname{Ind}(\mathcal{C})$. This construction for instance applies to the category of perfect complexes over some base, or to the category of spectra.

\section{Main results}

Before introducing a couple of examples that require this level of generality, let us state the main results. The goal is to construct invariants of Tate objects. To do so, we study the non-connective $\mathrm{K}$-theory spectrum $\mathbb{K}(\operatorname{Tate}(\mathcal{C}))$. Our next result gives an $\infty$-categorical analogue to a delooping result of Saito in [Sai], first conjectured by Kapranov and Previdi.

Theorem 1 (see Theorem 4.3). Let $\mathcal{C}$ be a stable and idempotent complete $(\infty, 1)$ category. The non-connective $K$-theory of $\operatorname{Tate}(\mathcal{C})$ is the suspension of that of $\mathcal{C}$ :

$$
\mathbb{K}(\operatorname{Tate}(\mathcal{C})) \simeq \Sigma \mathbb{K}(\mathcal{C}) .
$$

This theorem has two benefits: first, it shows that $\operatorname{Tate}(\mathcal{C})$ is a delooping for $\mathrm{K}$ theory. This kind of delooping are useful in order to grasp negative K-theory groups.

Secondly, Theorem 1 allows us to transfer invariants from objects of $\mathcal{C}$ to Tate objects. We apply this idea to algebraic geometry. We give a notion of sheaves of Tate complexes (i.e. Tate objects in perfect complexes). Given such a sheaf, we use Theorem 1 to define its dimensional torsor and its determinantal gerbe, two invariants obtained from the Euler characteristic and the determinant.

Theorem 2 (see Theorem 5.3). Let $\mathrm{K}^{\text {Tate }}$ denote the presheaf in spaces mapping $A$ to $\mathrm{K}(\operatorname{Tate}(\operatorname{Perf}(A)))$. Let $\mathrm{K}\left(\mathbb{G}_{m}, 2\right)$ denote the Eilenberg-MacLane classifying stack and let $\mathrm{B} \mathbb{Z}$ denote the stack classifying $\mathbb{Z}$-bundles. The determinant Det and the Euler characteristic Dim induce morphisms

$$
\begin{aligned}
{[\text { Det }]: \mathrm{K}^{\text {Tate }} } & \rightarrow \mathrm{K}\left(\mathbb{G}_{m}, 2\right), \\
{[\text { Dim }]: \mathrm{K}^{\text {Tate }} } & \rightarrow \mathrm{B} \mathbb{Z} .
\end{aligned}
$$

In particular, any Tate complex $E$ over some $X$ induces a determinantal class $\left[\operatorname{Det}_{E}\right] \in \mathrm{H}^{2}\left(X, \mathcal{O}_{X}^{\times}\right)$and a dimensional torsor $\left[\operatorname{Dim}_{E}\right] \in \mathrm{H}^{1}(X, \mathbb{Z})$. 


\section{Applications}

We give here a couple of applications. Those applications are beyond the scope of this article and we will not provide any proofs here. Most of the required proofs are in [Dri, Hen] or [FHK].

Tate objects naturally appear when considering local fields: the field of Laurent series $k((t))$ is a Tate vector space over $k$. The first application concerns the study of formal loop spaces, as defined in [KV1]. To any scheme $X$ of finite type, we associate its formal loop space: roughly speaking, it is a geometric object $\mathcal{L}^{1}(X)$ classifying maps from $\operatorname{Spec}(k((t)))$ to $X$. It has a natural Tate bundle (namely its tangent bundle) and using Theorem 2 we get a determinantal gerbe and a dimension torsor. For instance, when $X=\mathrm{B} \mathbb{G}_{m}$ is the classifying stack of line bundles, the dimension torsor encodes the natural valuation $k((t))^{\times} \rightarrow \mathbb{Z}$ while the determinantal gerbe corresponds to the Contou-Carrère symbol $k((t))^{\times} \times k((t))^{\times} \rightarrow k^{\times}$.

In $[\mathbf{H e n}]$, the author develops a higher dimensional analogue $\mathcal{L}^{d}(X)$ of the formal loop space. This new geometrical object classifies maps from a $d$-dimensional punctured formal neighbourhood $\operatorname{Spec}\left(k \llbracket t_{1}, \ldots, t_{d} \rrbracket\right) \backslash\{0\}$ to $X$. An algebraic version of Hartogs theorem forces us to works in the realm of derived algebraic geometry. Derived algebraic geometry is a context that allows to study some ill-behaved geometric situations (the most emblematic examples being non-generic intersections).

In this context, the tangent bundle of $\mathcal{L}^{d}(X)$ no longer lives in an exact category, but lives in the derived category of $\mathcal{L}^{d}(X)$, a stable $(\infty, 1)$-category. Exact categories are not enough here. In $[\mathbf{H e n}]$, we prove that in some cases the tangent of $\mathcal{L}^{d}(X)$ is a Tate complex. Our Theorem 2 then defines a determinantal anomaly for those higher dimensional formal loop spaces, generalising a result of [KV2]. In $[\mathbf{F H K}]$, Faonte, Kapranov and the author study the determinantal gerbe on $\mathcal{L}^{d}(X)$ when $X=\mathrm{B} G$ is the classifying stack of $G$-bundles. This gerbe is closely related to tame symbols in higher dimensions. Moreover, the tangent of the determinantal gerbe is a canonical (non-trivial) central extension of the derived Lie algebra $\mathbb{R} \Gamma\left(\operatorname{Spec}\left(k \llbracket t_{1}, \ldots, t_{d} \rrbracket\right) \backslash\{0\}, \mathfrak{g} \otimes \mathcal{O}\right)$. This extension is a higher dimensional analogue of the Kac-Moody algebras.

Although not yet fully explored, the dimensional torsor should also have some valuable applications. As aforementioned, it corresponds on $\mathcal{L}^{1}(X)$ to the valuation on $k((t))^{\times}$. In [Dri], Drinfeld suggests that in higher dimensions $d \geqslant 2$, the dimensional torsor on $\mathcal{L}^{d}(X)$ should play the role of the valuation. In particular, he suggests a construction of motivic integration in higher dimension, using this torsor.

Another possible source of examples is algebraic topology. If $X$ is a space, then the category of spectra over $X$ is a stable $(\infty, 1)$-category. Theorem 1 then gives a shifted version of Waldhausen's K-theory of $X$. Again, this example could not be studied using only exact categories.

\section{Outline of the paper}

In section 1 , we recall some useful facts about (stable) $(\infty, 1)$-categories.

In section 2 , we define the category of Tate objects $\operatorname{Tate}(\mathcal{C})$ in a stable $(\infty, 1)$ category $\mathcal{C}$ as the smallest full subcategory of $\operatorname{Pro} \operatorname{Ind}(\mathcal{C})$ which contains $\operatorname{Ind}(\mathcal{C})$ and $\operatorname{Pro}(\mathcal{C})$ and is stable and idempotent complete. We identify the universal property of Tate $(\mathcal{C})$ (see Theorem 2.7). 
In section 3 , we show that objects in $\operatorname{Tate}(\mathcal{C})$ are retracts of extensions $X^{p} \rightarrow$ $X \rightarrow X^{i}$ with $X^{p} \in \operatorname{Pro}(\mathcal{C})$ and $X^{i} \in \operatorname{Ind}(\mathcal{C})$ (see Theorem 3.4). In particular, the definition we gave in section 2 agrees with the intuitive one given in the introduction.

In section 4 , we prove Theorem 1 (see Theorem 4.3 below), and in section 5 we apply it to prove Theorem 2 (see Theorem 5.3 below).

\section{Related work}

Literature on Tate objects is flourishing. Let us cite here the work of Drinfeld [Dri], Previdi [Pre], Saito [Sai], Osipov and Zhu [OZ] and more recently Bräunling, Gröchenig and Wolfson [BGW]. The author has also been told that Barwick, Gröchenig and Wolfson are working on a theory of Tate objects in exact $(\infty, 1)$ categories.

\section{Acknowledgments}

The author would like to thank Bertrand Toën, Marco Robalo, Michael Gröchenig and Damien Calaque for the many discussions we had about the content of this article. He is also very grateful to the referee for the many valuable comments.

\section{Preliminaries}

This first section contains $\infty$-categorical preliminaries. Most of the content comes from $[\mathbf{H T T}]$. We will also define here some notation.

Notation Throughout this article, we will fix two universes $\mathbb{U} \in \mathbb{V}$.

Let us first set some notation, borrowed from [HTT]:

- We will denote by $\mathbf{C a t}_{\infty}^{\mathbb{U}}$ the $(\infty, 1)$-category of $\mathbb{U}$-small $(\infty, 1)$-categories - see [HTT, 3.0.0.1];

- Let $\operatorname{Pr}_{\infty}^{\mathrm{L}, \mathbb{U}}$ denote the $(\infty, 1)$-category of $\mathbb{U}$-presentable (and thus $\mathbb{V}$-small) $(\infty, 1)$-categories with left adjoint functors - see [HTT, 5.5.3.1];

- The symbol sSets will denote the $(\infty, 1)$-category of $\mathbb{U}$-small spaces;

- For any $(\infty, 1)$-categories $\mathcal{C}$ and $\mathcal{D}$ we will write $\operatorname{Fct}(\mathcal{C}, \mathcal{D})$ for the $(\infty, 1)$-category of functors from $\mathcal{C}$ to $\mathcal{D}$ (see [HTT, 1.2.7.3]). The category of presheaves will be denoted $\mathcal{P}(\mathcal{C})=\operatorname{Fct}\left(\mathcal{C}^{\text {op }}\right.$, sSets $)$.

- For any $(\infty, 1)$-category $\mathcal{C}$ and any objects $c$ and $d$ in $\mathcal{C}$, we will denote by $\operatorname{Map}_{\mathcal{C}}(c, d)$ the space of maps from $c$ to $d$.

The following theorem is a concatenation of results from Lurie.

Theorem 1.1 (Lurie). Let $\mathcal{C}$ be a $\mathbb{V}$-small $(\infty, 1)$-category. There is an $(\infty, 1)$-category $\operatorname{Ind}^{\mathbb{U}}(\mathcal{C})$ and a functor $j: \mathcal{C} \rightarrow \operatorname{Ind}^{\mathbb{U}}(\mathcal{C})$ such that

(i) The $(\infty, 1)$-category $\operatorname{Ind}^{\mathbb{U}}(\mathcal{C})$ is $\mathbb{V}$-small;

(ii) The $(\infty, 1)$-category $\mathbf{I n d}^{\mathbb{U}}(\mathcal{C})$ admits $\mathbb{U}$-small filtered colimits and is generated by $\mathbb{U}$-small filtered colimits of objects in $j(\mathcal{C})$;

(iii) The functor $j$ is fully faithful and preserves finite limits and finite colimits which exist in $\mathcal{C}$; 
(iv) For any $c \in \mathcal{C}$, its image $j(c)$ is $\mathbb{U}$-small compact in $\operatorname{Ind}^{\mathbb{U}}(\mathcal{C})$;

(v) For all $(\infty, 1)$-category $\mathcal{D}$ with every $\mathbb{U}$-small filtered colimits, the functor $j$ induces an equivalence

$$
\mathrm{Fct}^{\mathbb{U}-\mathrm{c}}\left(\operatorname{Ind}^{\mathbb{U}}(\mathcal{C}), \mathcal{D}\right) \stackrel{\sim}{\rightarrow} \operatorname{Fct}(\mathcal{C}, \mathcal{D}),
$$

where $\operatorname{Fct}^{\mathbb{U}-\mathrm{c}}\left(\operatorname{Ind}^{\mathbb{U}}(\mathcal{C}), \mathcal{D}\right)$ denotes the full subcategory of $\operatorname{Fct}\left(\operatorname{Ind}^{\mathbb{U}}(\mathcal{C}), \mathcal{D}\right)$ spanned by functors preserving $\mathbb{U}$-small filtered colimits;

(vi) If $\mathcal{C}$ is $\mathbb{U}$-small and admits all finite colimits then $\mathbf{I n d}^{\mathbb{U}}(\mathcal{C})$ is $\mathbb{U}$-presentable.

Proof. Let us use the notation of [HTT, 5.3.6.2]. Let $\mathcal{K}$ denote the collection of $\mathbb{U}$ small filtered simplicial sets. We then set $\operatorname{Ind}^{\mathbb{U}}(\mathcal{C})=\mathcal{P}_{\emptyset}^{\mathcal{K}}(\mathcal{C})$. Recall that $\operatorname{Ind}^{\mathbb{U}}(\mathcal{C})$ is then the full subcategory of $\mathcal{P}(\mathcal{C})$ generated by $\mathbb{U}$-small filtered colimits of diagrams in $\mathcal{C}$. It satisfies the required properties because of loc. cit. 5.3.6.2 and 5.5.1.1. We also need tiny modifications of the proofs of loc. cit. 5.3.5.14 and 5.3.5.5.

Lurie proved in [HTT, 5.3.5.15] that any map $c \rightarrow d \in \operatorname{Ind}^{\mathbb{U}}(\mathcal{C})$ is a colimit of a $\mathbb{U}$-small filtered diagram $K \rightarrow \operatorname{Fct}\left(\Delta^{1}, \mathcal{C}\right)$. We will need afterwards the following small refinement of this statement, inspired by [BGW, 3.9].

Proposition 1.2 (Strictification of morphisms). Let $\mathcal{C}$ be a $\mathbb{V}$-small $(\infty, 1)$-category. Let $f: c \rightarrow d$ be a morphism in Ind $^{\mathbb{U}}(\mathcal{C})$. Let also $\bar{c}: K \rightarrow \mathcal{C}$ and $\bar{d}: L \rightarrow \mathcal{C}$ be $\mathbb{U}$-small filtered diagrams of whom respectively $c$ and $d$ are colimits in $\operatorname{Ind}^{\mathbb{U}}(\mathcal{C})$. There exists $a \mathbb{U}$-small filtered diagram $\bar{f}: J \rightarrow \operatorname{Fct}\left(\Delta^{1}, \mathcal{C}\right)$ and a commutative diagram

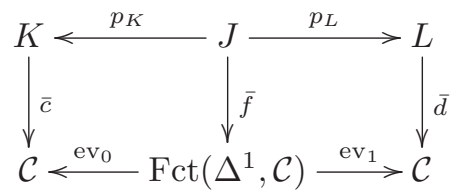

such that both maps $p_{K}$ and $p_{L}$ are cofinal, and such that $f$ is the colimit of $\bar{f}$.

In the proof of the above proposition, we will need the notation:

Definition 1.3 (see [HTT, 1.2.8.4]). Let $K$ be a simplicial set. We will denote by $K^{\triangleright}$ the simplicial set obtained from $K$ by formally adding a final object. This final object will be called the cone point of $K^{\triangleright}$.

Proof. Using [HTT, 4.3.2.14] we can assume that both $K$ and $L$ are filtered partially ordered sets. Let us denote by $J^{\prime}$ the fibre product

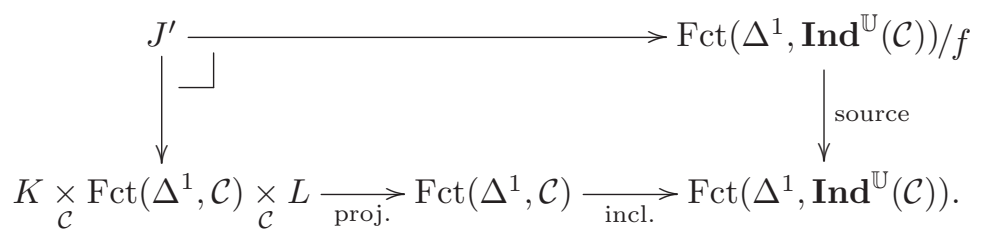

Let us first prove that $J^{\prime}$ is filtered. Let $P$ be a partially ordered finite set and $P^{\triangleright}$ denote the partially ordered set $P \cup\{\infty\}$, where $\infty$ is a maximal element. A morphism 
$P \rightarrow J^{\prime}$ is the datum of a commutative diagram

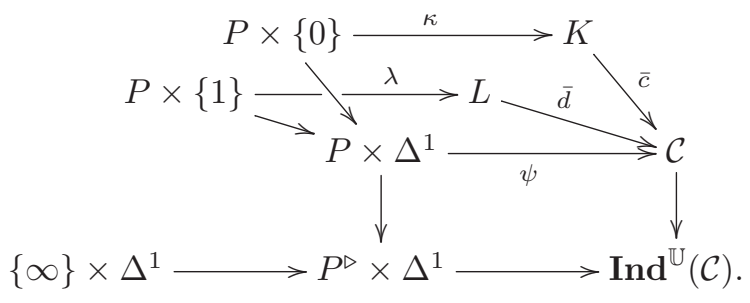

Let us denote by $P_{+}$the partially ordered set $P \cup\{+\}$ where + is a maximal element. Because $K$ is filtered, the map $\kappa$ extends to a morphism $\kappa^{\prime}: P_{+} \times\{0\} \rightarrow K$. There exists $l \in L$ such that the induced map $\bar{c}\left(\kappa^{\prime}(+)\right) \rightarrow c \rightarrow d$ factors through $\bar{d}(l) \rightarrow d$. Since $L$ is filtered, there is a map $\lambda^{\prime}: P_{+} \times\{1\} \rightarrow L$ extending $\lambda$. We can, moreover, chose $\lambda^{\prime}(+)$ greater than $l$ (i.e. with a map $l \rightarrow \lambda^{\prime}(+)$ in $L$ ). Using the map $\bar{c}\left(\kappa^{\prime}(+)\right) \rightarrow \bar{d}(l) \rightarrow \bar{d}\left(\lambda^{\prime}(+)\right)$, we get a morphism $\psi^{\prime}: P_{+} \times \Delta^{1} \rightarrow \mathcal{C}$ extending $\psi$, which by construction extends to $P_{+}^{\triangleright} \times \Delta^{1}$ - where we set $\infty \geqslant+$. This defines a morphism $P_{+} \rightarrow J^{\prime}$, proving that $J^{\prime}$ is filtered. Using [HTT, 4.3.2.14] we define $J$ to be a filtered partially ordered set with a cofinal map $J \rightarrow J^{\prime}$. Proving that the maps $J \rightarrow K$ and $J \rightarrow L$ are cofinal is now straightforward. This also implies that the induced diagram $\bar{f}: J \rightarrow \operatorname{Fct}\left(\Delta^{1}, \mathcal{C}\right)$ has colimit $f$ in $\operatorname{Ind}^{\mathbb{U}}(\mathcal{C})$.

Remark 1.4. Assume $\mathcal{C}$ admits finite colimits. We can consider the $\mathbb{V}$-presentable

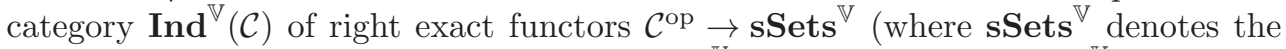
category of $\mathbb{V}$-small spaces). The category $\operatorname{Ind}^{\mathbb{U}}(\mathcal{C})$ then embeds in $\operatorname{Ind}^{\mathbb{V}}(\mathcal{C})$.

Definition 1.5. Let $\mathcal{C}$ be a $\mathbb{V}$-small $\infty$-category. We define $\operatorname{Pro}^{\mathbb{U}}(\mathcal{C})$ as the $(\infty, 1)$ category

$$
\operatorname{Pro}^{\mathbb{U}}(\mathcal{C})=\left(\operatorname{Ind}^{\mathbb{U}}\left(\mathcal{C}^{\mathrm{op}}\right)\right)^{\mathrm{op}}
$$

It satisfies properties dual to those of $\operatorname{Ind}^{\mathbb{U}}(\mathcal{C})$.

We will use extensively the notion of stable $(\infty, 1)$-categories. Those categories are the $\infty$-categorical analogues of triangulated categories (see [HAlg]).

Definition 1.6. A pointed $(\infty, 1)$-category $\mathcal{C}$ is stable if a square is cartesian if and only if it is cocartesian.

Let $\mathbf{C a t} t_{\infty}^{\mathbb{V} \text {,st }}$ denote the subcategory of $\mathbf{C a t}_{\infty}^{\mathbb{V}}$ spanned by stable categories with exact functors between them - see [HAlg, 1.1.4]. Let $\mathbf{C a t}_{\infty}^{\mathbb{V}, \text { st, id }}$ denote the full subcategory of $\mathbf{C a t}_{\infty}^{\mathbb{V} \text {,st }}$ spanned by idempotent complete stable categories.

Definition 1.7. Let $\mathcal{C} \in \mathbf{C a t}_{\infty}^{\mathbb{V} \text { st }}$. An exact sequence in $\mathcal{C}$ is a fibre (and hence also cofibre) sequence $X \rightarrow Y \rightarrow Z$ in $\mathcal{C}$.

The following lemma is a direct consequence of results from Lurie's [HTT].

Lemma 1.8 (Stable envelop). Let $\mathcal{C}$ be a $\mathbb{V}$-small pointed category with all suspensions. Let us assume that the suspension functor $\mathcal{C} \rightarrow \mathcal{C}$ is an equivalence. There exists an $(\infty, 1)$-category $\mathcal{C}^{\text {st }}$ with a map $j: \mathcal{C} \rightarrow \mathcal{C}^{\text {st }}$ such that

(i) The category $\mathcal{C}^{\text {st }}$ is $\mathbb{V}$-small and stable; 
(ii) The functor $j$ is fully faithful and preserves all limits and finite colimits which exist in $\mathcal{C}$;

(iii) For any stable $(\infty, 1)$-category $\mathcal{D}$ the induced map

$$
\operatorname{Fct}^{\mathrm{ex}}\left(\mathcal{C}^{\mathrm{st}}, \mathcal{D}\right) \rightarrow \operatorname{Fct}^{\text {lex }}(\mathcal{C}, \mathcal{D})
$$

between exact functors and left exact functors (i.e. preserving finite limits that exist in $\mathcal{C}$ ) is an equivalence;

(iv) For any stable category $\mathcal{D}$ with a fully faithful functor $\mathcal{C} \rightarrow \mathcal{D}$ preserving finite colimits and limits which exist in $\mathcal{C}$, the smallest stable subcategory of $\mathcal{D}$ containing the image of $\mathcal{C}$ is equivalent to $\mathcal{C}^{\text {st }}$.

Proof. Let us denote by $K$ the simplicial set corresponding to a diagram

Let $\mathcal{R}$ denote the collection of all cocartesian diagrams $K^{\triangleright} \rightarrow \mathcal{C}$ and the trivial diagram given by a zero object $\emptyset^{\triangleright}=\bullet \rightarrow \mathcal{C}$ in $\mathcal{C}$. We then set $\mathcal{C}^{\text {st }}=\mathcal{P}_{\mathcal{R}}^{\{K, \emptyset\}}(\mathcal{C})$ using the notation of [HTT, 5.3.6.2]. Note that (iii) is proven in loc. cit. The category $\mathcal{C}^{\text {st }}$ is pointed and it comes with two natural fully faithful maps

$$
\mathcal{C} \stackrel{j}{\longrightarrow} \mathcal{C}^{\text {st }} \longrightarrow \mathcal{P}(\mathcal{C})
$$

whose composite is the Yoneda functor and therefore preserves limits which exist in $\mathcal{C}$. It follows that $j$ also preserves those limits. By definition, the functor $j$ preserves finite colimits which exist in $\mathcal{C}$.

Any object of $\mathcal{C}^{\text {st }}$ is a finite colimit of objects in $\mathcal{C}$. Its suspension is therefore the colimit of the suspensions of those objects. The suspension functor $\mathcal{C}^{\text {st }} \rightarrow \mathcal{C}^{\text {st }}$ is thus an equivalence. Corollary [HAlg, 1.4.2.27] implies that $\mathcal{C}^{\text {st }}$ is stable.

We now focus on the assertion (iv). Let $f: \mathcal{C} \rightarrow \mathcal{D}$ be as required. Because of the third point, there is an essentially unique functor $g: \mathcal{C}^{\text {st }} \rightarrow \mathcal{D}$ lifting $f$. Every object in $\mathcal{C}^{\text {st }}$ can be written as both a colimit and a limit of objects of $\mathcal{C}$. It follows that $g$ is fully faithful and then that $\mathcal{C}^{\text {st }}$ contains the smallest full and stable subcategory $\mathcal{D}^{\prime}$ of $\mathcal{D}$ extending $\mathcal{C}$. There is also a universal map $\mathcal{C}^{\text {st }} \rightarrow \mathcal{D}^{\prime}$ which is easily seen to be an inverse to the inclusion.

Lemma 1.9. Let $\mathcal{C}$ be an idempotent complete $\mathbb{V}$-small $(\infty, 1)$-category. We consider the natural embeddings $i$ : $\operatorname{Pro}^{\mathbb{U}}(\mathcal{C}) \rightarrow \operatorname{Pro}^{\mathbb{U}} \operatorname{Ind}^{\mathbb{U}}(\mathcal{C})$ and $j: \operatorname{Ind}^{\mathbb{U}}(\mathcal{C}) \rightarrow \operatorname{Pro}^{\mathbb{U}} \operatorname{Ind}^{\mathbb{U}}(\mathcal{C})$. We will also denote by $k$ the embedding $\mathcal{C} \rightarrow \operatorname{Pro}^{\mathbb{U}} \mathbf{I n d}^{\mathbb{U}}(\mathcal{C})$. If an object of Pro $^{\mathbb{U}}$ Ind $^{\mathbb{U}}(\mathcal{C})$ is in both the essential images of $i$ and $j$, then it is in the essential image of $k$.

Proof. Let $x \in \mathbf{I n d}^{\mathbb{U}}(\mathcal{C})$. Let us assume there exists a pro-object $y \in \operatorname{Pro}^{\mathbb{U}}(\mathcal{C})$ and an equivalence $f: x \rightarrow y$. Let $\bar{y}: K^{\mathrm{op}} \rightarrow \mathcal{C}$ be a cofiltered diagram of whom $y$ is a limit in $\operatorname{Pro}^{\mathbb{U}}(\mathcal{C})$. The equivalence $f$ induces a morphism from the constant diagram $x: K^{\mathrm{op}} \rightarrow \mathbf{I n d}^{\mathbb{U}}(\mathcal{C})$ to $\bar{y}: K^{\mathrm{op}} \rightarrow \mathbf{I n d}^{\mathbb{U}}(\mathcal{C})$. An inverse $g: y \rightarrow x$ of $f$ then induces a map $y_{k}=\bar{y}(k) \rightarrow x$ for some $k \in K$ such that the composite morphism $x \rightarrow y_{k} \rightarrow x$ is homotopic to the identity. Idempotent completeness and [HTT, 5.4.2.4] finish the proof.

\section{Tate objects}

In this subsection we define the category of Tate objects in a stable $(\infty, 1)$-category. 
Definition 2.1. Let $\mathcal{C}$ be a $\mathbb{V}$-small stable $(\infty, 1)$-category. We define the category Tate $_{0}^{\mathbb{U}}(\mathcal{C})$ of pure Tate objects in $\mathcal{C}$ as the full sub-category of $\mathbf{P r o}^{\mathbb{U}} \mathbf{I n d}^{\mathbb{U}}(\mathcal{C})$ spanned by the images of $\operatorname{Ind}^{\mathbb{U}}(\mathcal{C})$ and $\operatorname{Pro}^{\mathbb{U}}(\mathcal{C})$ through the canonical functors. The category Tate $_{0}^{\mathbb{U}}(\mathcal{C})$ obviously satisfies the conditions of Theorem 1.8 and we define the category Tate $_{\mathrm{el}}^{\mathbb{U}}(\mathcal{C})$ of elementary Tate objects in $\mathcal{C}$ as the stable envelop

$$
\operatorname{Tate}_{\mathrm{el}}^{\mathbb{U}}(\mathcal{C})=\left(\operatorname{Tate}_{0}^{\mathbb{U}}(\mathcal{C})\right)^{\mathrm{st}} .
$$

We also define the category $\operatorname{Tate}^{\mathbb{U}}(\mathcal{C})$ of Tate objects in $\mathcal{C}$ as the idempotent completion of Tate $_{\mathrm{el}}^{\mathbb{U}}(\mathcal{C})$. We have fully faithful exact functors between stable $(\infty, 1)$ categories

$$
\operatorname{Tate}_{\mathrm{el}}^{\mathbb{U}}(\mathcal{C}) \rightarrow \operatorname{Tate}^{\mathbb{U}}(\mathcal{C}) \rightarrow \text { Pro }^{\mathbb{U}} \operatorname{Ind}^{\mathbb{U}}(\mathcal{C})
$$

Remark 2.2. It follows from Theorem 1.8 that $\operatorname{Tate}_{\mathrm{el}}^{\mathbb{U}}(\mathcal{C})\left(\operatorname{resp}\right.$. $\operatorname{Tate}^{\mathbb{U}}(\mathcal{C})$ ) is the smallest stable (resp. stable and idempotent complete) subcategory of Pro ${ }^{\mathbb{U}} \operatorname{Ind}^{\mathbb{U}}(\mathcal{C})$ containing both the essential images of $\operatorname{Ind}^{\mathbb{U}}(\mathcal{C})$ and $\operatorname{Pro}^{\mathbb{U}}(\mathcal{C})$. We will see (Theorem 2.8) that the same holds in $\operatorname{Ind}^{\mathbb{U}} \operatorname{Pro}^{\mathbb{U}}(\mathcal{C})$ instead of $\operatorname{Pro}^{\mathbb{U}} \operatorname{Ind}^{\mathbb{U}}(\mathcal{C})$.

Remark 2.3. In Theorem 2.1 as well as in all the results below, the role of the universe $\mathbb{U}$ may be played by any cardinal $\kappa$. Replacing $\operatorname{Ind}^{\mathbb{U}}(\mathcal{C})\left(\operatorname{resp} . \operatorname{Pro}^{\mathbb{U}}(\mathcal{C})\right)$ by the categories of $\kappa$-compact (resp. cocompact) objects. We would require $\kappa$ to be regular for Theorem 3.17 (and hence Theorem 3.16) to hold.

Example 2.4. Let $\mathcal{C}$ be the category of perfect complexes over a field $k$. There is a natural incarnation of $k((t))$ in $\operatorname{Tate}^{\mathbb{U}}\left(\operatorname{Perf}_{k}\right)$, given by the isomorphism (of vector spaces)

$$
k((t)) \simeq \lim _{p} \operatorname{colim}_{n} t^{-n} k[t] / t^{p+n} .
$$

To see it actually lives in Tate objects, one can write the isomorphism $k((t)) \simeq k \llbracket t \rrbracket \oplus$ $t^{-1} k\left[t^{-1}\right]$ and translate it in terms of objects in $\mathbf{P r o}^{\mathbb{U}} \mathbf{I n d}^{\mathbb{U}}\left(\mathbf{P e r f}_{k}\right)$. It follows that $\lim _{p} \operatorname{colim}_{n} t^{-n} k[t] / t^{p+n}$ indeed lies in $\operatorname{Tate}^{\mathbb{U}}\left(\boldsymbol{P e r f}_{k}\right)$. We will see in section 3 that any Tate object can be written as such an extension, of an ind-object by a pro-object.

The above example does not require $(\infty, 1)$-categories to work. Although, it leads to the following generalisation. We can see $k((t))$ as the ring of functions on the punctured formal neighbourhood $\widehat{\mathbb{A}}^{1} \backslash\{0\}$. Now considering the complex of derived global sections of the sheaf of functions on the punctured formal neighbourhood $\widehat{\mathbb{A}}^{d} \backslash\{0\}=\operatorname{Spec}\left(k \llbracket t_{1}, \ldots, t_{d} \rrbracket\right) \backslash\{0\}$ of dimension $d$. Computing its cohomology, we get for $d \geqslant 2$

$$
\mathrm{H}^{n}\left(\widehat{\mathbb{A}}^{d} \backslash\{0\}, \mathcal{O}\right) \simeq \begin{cases}k \llbracket t_{1}, \ldots, t_{d} \rrbracket & \text { if } n=0, \\ \left(t_{1} \ldots t_{d}\right)^{-1} k\left[t_{1}^{-1}, \ldots, t_{d}^{-1}\right] & \text { if } n=d-1 \\ 0 & \text { else. }\end{cases}
$$

Hence we get $\mathbb{R} \Gamma\left(\widehat{\mathbb{A}}^{d} \backslash\{0\}, \mathcal{O}\right) \simeq k \llbracket t_{1}, \ldots, t_{d} \rrbracket \oplus\left(t_{1} \ldots t_{d}\right)^{-1} k\left[t_{1}^{-1}, \ldots, t_{d}^{-1}\right][1-d]$ (where $[1-d]$ is the shift by $1-d$ ). It is again the extension of a pro-object by an ind-object. 
Remark 2.5. Note that $\operatorname{Tate}^{\mathbb{U}}(\mathcal{C})$ is $\mathbb{V}$-small. The construction $\operatorname{Tate}^{\mathbb{U}}(-)$ defines a functor

$$
\mathrm{Cat}_{\infty}^{\mathbb{V}, \mathrm{st}} \rightarrow \mathrm{Cat}_{\infty}^{\mathbb{V}, \mathrm{st}, \mathrm{id}}
$$

It comes with a fully faithful - i.e. pointwise fully faithful - natural transformation

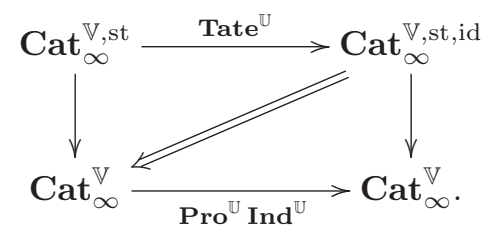

Remark 2.6. We can immediately see that the functor Tate ${ }^{\mathbb{U}}$ map any fully faithful and exact functor $\mathcal{C} \rightarrow \mathcal{D}$ between stable categories to a fully faithful (and exact) functor $\operatorname{Tate}^{\mathbb{U}}(\mathcal{C}) \rightarrow \operatorname{Tate}^{\mathbb{U}}(\mathcal{D})$.

Let us now give a universal property for the category of pure Tate objects. The next theorem states that for any $(\infty, 1)$-category $\mathcal{D}$ and any commutative diagram

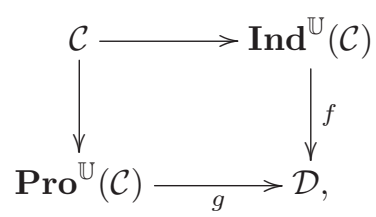

such that $f$ preserves $\mathbb{U}$-small filtered colimits and $g$ preserves $\mathbb{U}$-small cofiltered limits there exists an essentially unique functor $\operatorname{Tate}_{0}^{\mathbb{U}}(\mathcal{C}) \rightarrow \mathcal{D}$ such that $f$ and $g$ are respectively equivalent to the composite functors

$$
\begin{aligned}
& \operatorname{Ind}^{\mathbb{U}}(\mathcal{C}) \rightarrow \operatorname{Tate}_{0}^{\mathbb{U}}(\mathcal{C}) \rightarrow \mathcal{D}, \\
& \operatorname{Pro}^{\mathbb{U}}(\mathcal{C}) \rightarrow \operatorname{Tate}_{0}^{\mathbb{U}}(\mathcal{C}) \rightarrow \mathcal{D} .
\end{aligned}
$$

This universal property was discovered during a discussion with Michael Gröchenig, whom the author thanks greatly. To state formally this property, let us fix some notation. Let $i: \operatorname{Ind}^{\mathbb{U}}(\mathcal{C}) \rightarrow$ Tate $_{0}^{\mathbb{U}}(\mathcal{C})$ and $p: \operatorname{Pro}^{\mathbb{U}}(\mathcal{C}) \rightarrow$ Tate $_{0}^{\mathbb{U}}(\mathcal{C})$ denote the canonical inclusions. We will denote by $\operatorname{Fct}_{t}\left(\operatorname{Tate}_{0}^{\mathbb{U}}(\mathcal{C}), \mathcal{D}\right)$ the full subcategory of $\operatorname{Fct}\left(\operatorname{Tate}_{0}^{\mathbb{U}}(\mathcal{C}), \mathcal{D}\right)$ spanned by those functors $\xi$ such that

- The composite functor $\xi i$ maps filtered colimit diagrams to colimit diagrams.

- The composite functor $\xi p$ maps cofiltered limit diagrams to limit diagrams.

Let also $\operatorname{Fct}_{m}(\mathcal{C}, \mathcal{D})$ denote the category of functors $g: \mathcal{C} \rightarrow \mathcal{D}$ such that

- For any filtered diagram $K \rightarrow \mathcal{C}$, the composite diagram $K \rightarrow \mathcal{C} \rightarrow \mathcal{D}$ admits a colimit in $\mathcal{D}$.

- For any cofiltered diagram $K^{\mathrm{op}} \rightarrow \mathcal{C}$, the composite diagram $K^{\mathrm{op}} \rightarrow \mathcal{C} \rightarrow \mathcal{D}$ admits a limit in $\mathcal{D}$.

Theorem 2.7. Let $\mathcal{C}$ be a $\mathbb{V}$-small stable $(\infty, 1)$-category. For any $(\infty, 1)$-category $\mathcal{D}$, the restriction functor induces an equivalence

$$
\operatorname{Fct}_{t}\left(\operatorname{Tate}_{0}^{\mathbb{U}}(\mathcal{C}), \mathcal{D}\right) \longrightarrow \operatorname{Fct}_{m}(\mathcal{C}, \mathcal{D}) .
$$


Proof. Let us shorten the notation:

$$
\mathrm{IC}=\operatorname{Ind}^{\mathbb{U}}(\mathcal{C}), \quad \mathrm{PC}=\operatorname{Pro}^{\mathbb{U}}(\mathcal{C}), \quad \mathrm{TC}=\operatorname{Tate}_{0}^{\mathbb{U}}(\mathcal{C}), \quad \operatorname{PIC}=\operatorname{Pro}^{\mathbb{U}}\left(\operatorname{Ind}^{\mathbb{U}}(\mathcal{C})\right)
$$

Recall that $\mathcal{P}(\mathcal{D})$ denotes the $(\infty, 1)$-category of simplicial presheaves on $\mathcal{D}$. The restriction functor $\mathrm{Fct}(\mathrm{PIC}, \mathcal{P}(\mathcal{D})) \rightarrow \operatorname{Fct}(\mathrm{TC}, \mathcal{P}(\mathcal{D}))$ admits a left adjoint given by the left Kan extension. The restriction functor $\operatorname{Fct}(\mathrm{PIC}, \mathcal{P}(\mathcal{D})) \rightarrow \operatorname{Fct}(\mathrm{IC}, \mathcal{P}(\mathcal{D}))$ admits a right adjoint, given by the right Kan extension. Let us fix their notation

$$
\operatorname{Fct}(\mathrm{TC}, \mathcal{P}(\mathcal{D})) \underset{\gamma}{\stackrel{\delta}{\gtrless}} \operatorname{Fct}(\operatorname{PIC}, \mathcal{P}(\mathcal{D})) \underset{\alpha}{\stackrel{\beta}{\gtrless}} \operatorname{Fct}(\mathrm{IC}, \mathcal{P}(\mathcal{D}))
$$

the left adjoints being represented above their right adjoint. Note that both $\alpha$ and $\delta$ are fully faithful. Let also $\tau$ denote the fully faithful functor

$$
\begin{aligned}
\operatorname{Fct}_{m}(\mathcal{C}, \mathcal{D}) \simeq \operatorname{Fct}^{\mathrm{c}}(\mathrm{IC}, \mathcal{D}) & \times \operatorname{Fct}^{\mathrm{l}}(\mathrm{PC}, \mathcal{D}) \\
\operatorname{Fct}(\mathcal{C}, \mathcal{D}) & \downarrow_{\tau} \\
& \operatorname{Fct}(\mathrm{IC}, \mathcal{P}(\mathcal{D})) \times \operatorname{Fct}^{\mathrm{l}}(\mathrm{PC}, \mathcal{P}(\mathcal{D})) \simeq \operatorname{Fct}(\mathrm{IC}, \mathcal{P}(\mathcal{D})),
\end{aligned}
$$

where $\mathrm{Fct}^{\mathrm{c}}$ (resp. Fct ${ }^{\mathrm{l}}$ ) denotes the category of functors preserving filtered colimits (resp. cofiltered limits) which exist in the source. We use here that the Yoneda embedding $\mathcal{D} \rightarrow \mathcal{P}(\mathcal{D})$ preserves limits. Let $\theta$ be the fully faithful functor

$$
\operatorname{Fct}_{t}(\mathrm{TC}, \mathcal{D}) \stackrel{\theta}{\longrightarrow} \operatorname{Fct}(\mathrm{TC}, \mathcal{P}(\mathcal{D}))
$$

The composite functor $\beta \delta$ is nothing but the restriction along the canonical inclusion $\mathrm{IC} \rightarrow \mathrm{TC}$. It follows that $\beta \delta \theta$ has image in the essential image of $\tau$. On the other hand, the functor $\gamma \alpha \tau$ has image in the essential image of $\theta$. We hence get an adjunction

$$
f: \operatorname{Fct}_{t}(\mathrm{TC}, \mathcal{D}) \rightleftarrows \operatorname{Fct}_{m}(\mathcal{C}, \mathcal{D}): g,
$$

where $f$ is left adjoint to $g$. The functor $g$ is equivalent to the restriction functor and the unit transformation $f g \rightarrow \operatorname{id}_{X}$ is then an equivalence. Moreover, as objects of $\mathrm{TC}$ are either pro-objects or ind-objects, the restriction functor $f$ is conservative. It follows that the above adjunction is an equivalence.

Corollary 2.8. The category of Tate objects is equivalent to the smallest stable and idempotent complete full subcategory of $\operatorname{Ind}^{\mathbb{U}} \operatorname{Pro}^{\mathbb{U}}(\mathcal{C})$ generated by the images of $\operatorname{Ind}^{\mathbb{U}}(\mathcal{C})$ and $\operatorname{Pro}^{\mathbb{U}}(\mathcal{C})$.

Proof. This follows from Theorem 2.7 and Theorem 1.8 .

Remark 2.9. The fully faithful functor $\mathrm{j}: \operatorname{Tate}_{0}^{\mathbb{U}}(\mathcal{C}) \rightarrow \mathbf{P r o}^{\mathbb{U}} \mathbf{I n d}^{\mathbb{U}}(\mathcal{C})$ preserves both the limits and colimits which exist in $\operatorname{Tate}_{0}^{\mathbb{U}}(\mathcal{C})$. Let indeed $\bar{x}: K \rightarrow$ Tate $_{0}^{\mathbb{U}}(\mathcal{C})$ be a diagram which admits a colimit $x \in \mathbf{T a t e}_{0}^{\mathbb{U}}(\mathcal{C})$. Let us denote by $x^{\prime}$ a colimit of $\mathrm{j} \bar{x}$ in 
Pro $^{\mathbb{U}}$ Ind $^{\mathbb{U}}(\mathcal{C})$. We have, for any cofiltered diagram $y: L^{\mathrm{op}} \rightarrow \operatorname{Ind}^{\mathbb{U}}(\mathcal{C})$

$$
\begin{aligned}
\operatorname{Map}_{\mathbf{P r o}^{\mathbb{U}} \mathbf{I n d}^{\mathbb{U}}(\mathcal{C})}\left(x^{\prime}, \lim \bar{y}\right) & \simeq \underset{l}{\operatorname{colim}} \operatorname{colim}_{k} \operatorname{Map}_{\mathbf{P r o}^{\mathbb{U}} \mathbf{I n d}^{\mathbb{U}}(\mathcal{C})}(\mathrm{j} \bar{x}, \bar{y}) \\
& \simeq \operatorname{colim}_{l} \operatorname{colim}_{k} \operatorname{Map}_{\mathbf{T a t e}_{0}^{\mathbb{U}}(\mathcal{C})}(\bar{x}, \bar{y}) \\
& \simeq \operatorname{colim}_{l} \operatorname{Map}_{\mathbf{T a t e}_{0}^{\mathbb{U}}(\mathcal{C})}(x, \bar{y}) \simeq \operatorname{colim}_{l} \operatorname{Map}_{\mathbf{P r o}^{\mathbb{U}} \mathbf{I n d}^{\mathbb{U}}(\mathcal{C})}(x, \bar{y}) \\
& \simeq \operatorname{Map}_{\mathbf{P r o}^{\mathbb{U}} \mathbf{I n d}^{\mathbb{U}}(\mathcal{C})}(x, \lim \bar{y}) .
\end{aligned}
$$

We show symmetrically that the inclusion $\operatorname{Tate}_{0}^{\mathbb{U}}(\mathcal{C}) \rightarrow \operatorname{Ind}^{\mathbb{U}}$ Pro $^{\mathbb{U}}(\mathcal{C})$ preserves limits. It follows that limits and colimits that exist in Tate $_{0}^{\mathbb{U}}(\mathcal{C})$ are exactly those coming from diagram in either $\operatorname{Ind}^{\mathbb{U}}(\mathcal{C})$ or $\operatorname{Pro}^{\mathbb{U}}(\mathcal{D})$. We can hence reformulate the universal property from Theorem 2.7 as follows: The datum of a commutative square

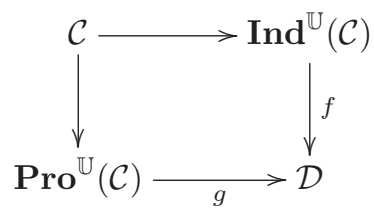

such that $f$ preserves filtered colimits and $g$ preserves cofiltered limits is equivalent to that of a functor Tate $_{0}^{\mathbb{U}}(\mathcal{C}) \rightarrow \mathcal{D}$ preserving both filtered colimits and cofiltered limits which exist in $\operatorname{Tate}_{0}^{\mathbb{U}}(\mathcal{C})$.

Let us close this section with the following lemma.

Lemma 2.10. Let $\mathcal{C}$ be $a \mathbb{V}$-small stable $(\infty, 1)$-category with a functor $f: \mathcal{C}^{\mathrm{op}} \rightarrow \mathcal{C}$. The functor $f$ induces a functor

$$
\tilde{f}:\left(\operatorname{Pro}^{\mathbb{V}} \operatorname{Ind}^{\mathbb{U}}(\mathcal{C})\right)^{\text {op }} \rightarrow \text { Pro }^{\mathbb{V}} \operatorname{Ind}^{\mathbb{U}}(\mathcal{C})
$$

which maps (elementary) $\mathbb{U}$-Tate objects to (elementary) $\mathbb{U}$-Tate objects.

If, moreover, the functor $f$ is an equivalence, then $\tilde{f}$ induces an equivalence

$$
\left(\operatorname{Tate}^{\mathbb{U}}(\mathcal{C})\right)^{\text {op }} \simeq \operatorname{Tate}^{\mathbb{U}}(\mathcal{C})
$$

Remark 2.11. The above lemma applies for instance when $\mathcal{C}$ is the category of perfect complexes on a base $k$. The duality functor $(-)^{\vee}=\underline{\operatorname{Hom}}(-, k)$ induces a duality of Tate objects

$$
(-)^{\vee}: \text { Tate }_{k}=\operatorname{Tate}\left(\operatorname{Perf}_{k}\right) \stackrel{\sim}{\rightarrow} \text { Tate }_{k}^{\mathrm{op}} .
$$

In particular, for any Tate object $X$, we have $\left(X^{\vee}\right)^{\vee} \simeq X$.

Proof. The category Pro ${ }^{\mathbb{V}} \mathbf{I n d}^{\mathbb{U}}(\mathcal{C})$ has all $\mathbb{V}$-small limits and colimits - it is the opposite category of a $\mathbb{V}$-presentable category. We define the functor $\tilde{f}$ as the extension of the composition

$$
\mathcal{C}^{\mathrm{op}} \rightarrow \mathcal{C} \rightarrow \text { Pro }^{\mathbb{V}} \operatorname{Ind}^{\mathbb{U}}(\mathcal{C})
$$

It maps objects of $\operatorname{Ind}^{\mathbb{U}}(\mathcal{C})$ to objects of $\operatorname{Pro}^{\mathbb{U}}(\mathcal{C}) \subset$ Pro $^{\mathbb{V}}(\mathcal{C})$ and vice-versa and therefore preserves pure Tate objects. The functor $\tilde{f}$ also preserves finite limits. It follows that it preserves Tate objects. 


\section{Lattices}

Tate object are characterised by the existence of a lattice. A lattice for a pro-indobject $X$ is an exact sequence

$$
X^{p} \rightarrow X \rightarrow X^{i}
$$

where $X^{p}$ is a pro-object and $X^{i}$ is an ind-object. We will see below that a pro-indobject is an elementary Tate object if and only if it admits a lattice. We will then study the category of lattices of a given Tate object.

Proposition 3.1. Let $\mathcal{C}$ be $a \mathbb{V}$-small stable $(\infty, 1)$-category. For any elementary Tate objects $X \in$ Tate $_{\mathrm{el}}^{\mathbb{U}}(\mathcal{C})$ there exists a $\mathbb{U}$-small cofiltered diagram $\bar{X}: K^{\mathrm{op}} \rightarrow \operatorname{Ind}^{\mathbb{U}}(\mathcal{C})$ such that

- The object $X$ is a limit of $\bar{X}$ in $\operatorname{Pro}^{\mathbb{U}} \operatorname{Ind}^{\mathbb{U}}(\mathcal{C})$ and

- For any $k \in K$ the diagram $\operatorname{ker}(\bar{X} \rightarrow \bar{X}(k)):(k / K)^{\mathrm{op}} \rightarrow \operatorname{Ind}^{\mathbb{U}}(\mathcal{C})$ has values in the essential image of $\mathcal{C}$.

Definition 3.2. Let $\mathcal{C}$ be a $\mathbb{V}$-small stable $(\infty, 1)$-category. For any elementary Tate object $X \in$ Tate $_{\text {el }}^{\mathbb{U}}(\mathcal{C})$, we will call a Tate diagram for $X$ any $\mathbb{U}$-small cofiltered diagram $\bar{X}: K^{\text {op }} \rightarrow$ Ind $^{\mathbb{U}}(\mathcal{C})$ as in Theorem 3.1.

of Theorem 3.1. Let $\mathcal{D}$ denote the full subcategory of $\mathbf{P r o}^{\mathbb{U}} \mathbf{I n d}^{\mathbb{U}}(\mathcal{C})$ spanned by those objects $X$ satisfying the conclusion of the proposition. The category $\mathcal{D}$ obviously contains both the essential images of $\operatorname{Ind}^{\mathbb{U}}(\mathcal{C})$ and Pro ${ }^{\mathbb{U}}(\mathcal{C})$. It suffices to prove that $\mathcal{D}$ is stable by extension. We see that it is stable by shifts and we can thus consider an exact sequence $X \rightarrow X_{0} \rightarrow X_{1}$ in $\mathbf{P r o}^{\mathbb{U}}$ Ind $^{\mathbb{U}}(\mathcal{C})$ such that both $X_{0}$ and $X_{1}$ are in $\mathcal{D}$. Let $\bar{X}_{0}: K^{\mathrm{op}} \rightarrow \operatorname{Ind}^{\mathbb{U}}(\mathcal{C})$ and $\bar{X}_{1}: L^{\mathrm{op}} \rightarrow \operatorname{Ind}^{\mathbb{U}}(\mathcal{C})$ be Tate diagrams for $X_{0}$ and $X_{1}$ respectively. Using Theorem 1.2 , we can assume $K=L$ and that we have a diagram $K^{\mathrm{op}} \rightarrow \operatorname{Fct}\left(\Delta^{1}, \operatorname{Ind}^{\mathbb{U}}(\mathcal{C})\right)$ of whom the map $X_{0} \rightarrow X_{1}$ is a limit. Considering the pointwise kernel, we get a diagram $\bar{X}: K^{\text {op }} \rightarrow \operatorname{Ind}^{\mathbb{U}}(\mathcal{C})$ of whom $X$ is a limit. It obviously satisfies the required property.

Remark 3.3. To state the above proposition in an informal way, any elementary Tate object $X$ can be represented by a diagram $\lim _{\alpha} \operatorname{colim}_{\beta} X_{\alpha \beta}$ such that for any $\alpha_{0}$, the kernel of canonical projection $X \rightarrow \operatorname{colim}_{\beta} X_{\alpha_{0} \beta}$ is actually a pro-object. This obviously imply the following corollary.

Corollary 3.4. Any elementary Tate object $X$ fits into an exact sequence

$$
X^{p} \rightarrow X \rightarrow X^{i}
$$

where $X^{p} \in \operatorname{Pro}^{\mathbb{U}}(\mathcal{C})$ and $X^{i} \in \operatorname{Ind}^{\mathbb{U}}(\mathcal{C})$.

Definition 3.5. Let $\mathcal{C}$ be a $\mathbb{V}$-small stable $(\infty, 1)$-category. For any elementary Tate object $X \in$ Tate $_{\mathrm{el}}^{\mathbb{U}}(\mathcal{C})$, we will call a lattice of $X$ any exact sequence

$$
X^{p} \rightarrow X \rightarrow X^{i}
$$

where $X^{p} \in \operatorname{Pro}^{\mathbb{U}}(\mathcal{C})$ and $X^{i} \in \operatorname{Ind}^{\mathbb{U}}(\mathcal{C})$.

Remark 3.6. Let $X$ be an elementary Tate object in $\mathcal{C}$. Let us consider a lattice $X^{p} \rightarrow$ $X \rightarrow X^{i}$ of $X$. We will construct a Tate diagram for $X$ out of it. Let $\bar{X}^{p}: K^{\text {op }} \rightarrow \mathcal{C}$ 


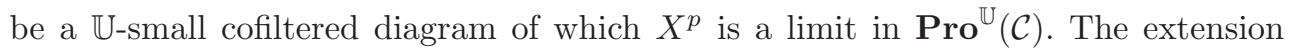
map $X^{i}[-1] \rightarrow X^{p}$ induces a natural transformation $X^{i}[-1] \rightarrow \bar{X}^{p}$ from the constant diagram $X^{i}[-1]: K^{\mathrm{op}} \rightarrow \operatorname{Ind}^{\mathbb{U}}(\mathcal{C})$ to $\bar{X}^{p}$. The quotient of this natural transformation defines a diagram $\bar{X}: K^{\text {op }} \rightarrow$ Ind $^{\mathbb{U}}(\mathcal{C})$ which is by construction a Tate diagram for $X$ : for any morphism $k \rightarrow l$ in $K$, the kernel of the induced map $\bar{X}(l) \rightarrow \bar{X}(k)$ is equivalent to that of $\bar{X}^{p}(l) \rightarrow \bar{X}^{p}(k)$ which belongs to $\mathcal{C}$.

Remark 3.7. In the literature, the word "lattice" is often dedicated to maps $X^{p} \rightarrow X$ whose quotient is an ind-object - where $X^{p}$ is a pro-object.

Lemma 3.8. Let $\mathcal{C}$ be a $\mathbb{V}$-small stable $(\infty, 1)$-category. Let $f: X \rightarrow Y$ be a map between elementary Tate objects in $\mathcal{C}$. For any lattice $Y^{p} \rightarrow Y \rightarrow Y^{i}$ there exists a lattice $X^{p} \rightarrow X \rightarrow X^{i}$ compatible with $f$, i.e. fitting in a commutative diagram

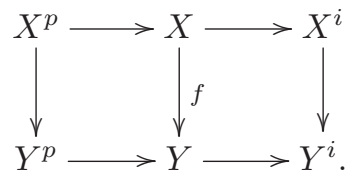

Dually, for any lattice $X^{p} \rightarrow X \rightarrow X^{i}$ there exists a lattice $Y^{p} \rightarrow Y \rightarrow Y^{i}$ and a commutative diagram as above.

Remark 3.9. In particular, any map $X \rightarrow Y^{i}$ from a Tate object to an ind-object factors through a lattice $X \rightarrow X^{i}$ of $X$.

Proof. Choose any lattice $X_{0}^{p} \rightarrow X \rightarrow X_{0}^{i}$ for $X$. The composite map $X_{0}^{p} \rightarrow X \stackrel{f}{\rightarrow}$ $Y \rightarrow Y^{i}$ goes from a pro-object to an ind-object. It therefore factors through an object $E \in \mathcal{C}$. We define $X^{p}$ as the fibre of the map $X_{0}^{p} \rightarrow E$ and $X^{i}$ as the cofibre of the map $X^{p} \rightarrow X$. By construction, we have $X^{p} \in \operatorname{Pro}^{\mathbb{U}}(\mathcal{C})$ and a commutative diagram

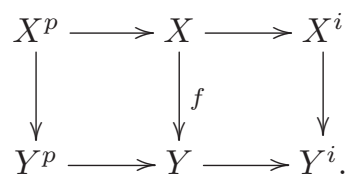

Moreover, the fibre of the induced map $X^{i} \rightarrow X_{0}^{i}$ is equivalent to $E$ and thus $X^{i} \in$ $\operatorname{Ind}^{\mathbb{U}}(\mathcal{C})$.

Let us now denote by $\operatorname{Latt}_{\mathcal{C}}$ the full-subcategory of $\operatorname{Fct}\left(\Delta^{1} \times \Delta^{1}, \operatorname{Pro}^{\mathbb{U}} \operatorname{Ind}^{\mathbb{U}}(\mathcal{C})\right)$ spanned by the cocartesian (and hence also cartesian) squares of the form

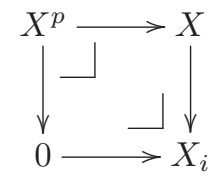

where $X^{p}$ lies in the essential image of $\operatorname{Pro}^{\mathbb{U}}(\mathcal{C})$ and $X^{i}$ lies in that of $\operatorname{Ind}^{\mathbb{U}}(\mathcal{C})$. Let us denote by $q$ the natural functor $\operatorname{Latt}_{\mathcal{C}} \rightarrow$ Tate $_{\mathrm{el}}^{\mathbb{U}}(\mathcal{C})$ mapping a square as above to $X$. Let us also denote the $\pi^{p}$ and $\pi_{i}$ the natural functors from $\operatorname{Latt}_{\mathcal{C}}$ to $\operatorname{Pro}^{\mathbb{U}}(\mathcal{C})$ and $\operatorname{Ind}^{\mathbb{U}}(\mathcal{C})$ respectively. 
Definition 3.10. For any elementary Tate object $X$, we will $\operatorname{denote}_{\text {by }} \operatorname{Latt}_{\mathcal{C}}(X)$ the fibre category $q^{-1}(X)$. We will call it the category of lattices of $X$.

Remark 3.11. In the literature, the category $\operatorname{Latt}_{\mathcal{C}}(X)$ defined above is sometimes called the Sato Grassmannian of $X$.

Let us study morphisms between lattices. Note first that a lattice $X^{p} \rightarrow X \rightarrow X^{i}$ is determined by the morphism $X^{p} \rightarrow X$. A map between lattices is a commutative diagram

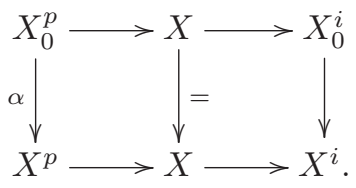

It is actually determined by the quotient of $\alpha$, which belongs to $\mathcal{C}$. That is what the following lemma is about.

Lemma 3.12. Let $X^{\bullet}=\left(X^{p} \rightarrow X \rightarrow X^{i}\right)$ be a lattice. There is a canonical equivalence

$$
\operatorname{Latt}_{\mathcal{C}}(X) / X^{\bullet} \simeq X^{p} / \mathcal{C}
$$

Proof. We consider the inclusion $\{(0,0)\} \rightarrow \Delta^{1} \times \Delta^{1}$. It induces a functor $\operatorname{Latt}_{\mathcal{C}} \rightarrow$ $\operatorname{Pro}^{\mathbb{U}}(\mathcal{C})$. In particular, we get

$$
P: \operatorname{Latt}_{\mathcal{C}}(X) / X^{\bullet} \rightarrow \operatorname{Pro}^{\mathbb{U}}(\mathcal{C}) / X^{p}
$$

Remark that the functor $P$ is fully faithful (by essential uniqueness of cofibres and compositions). Let us consider a morphism of lattices of $X$

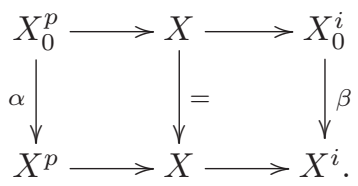

The quotient $x$ of $\alpha$ is equivalent to the shift of that of $\beta$. It follows from Theorem 1.9 that $x$ belongs to the essential image of $\mathcal{C}$. The composite functor

$$
\operatorname{Latt}_{\mathcal{C}}(X) / X^{\bullet} \stackrel{P}{\longrightarrow} \operatorname{Pro}^{\mathbb{U}}(\mathcal{C}) / X^{p} \stackrel{Q}{\sim} X^{p} / \operatorname{Pro}^{\mathbb{U}}(\mathcal{C}),
$$

therefore, has image in the full subcategory $X^{p} / \mathcal{C}$. We get a fully faithful functor

$$
\phi: \operatorname{Latt}_{\mathcal{C}}(X) / X^{\bullet} \rightarrow X^{p} / \mathcal{C}
$$

Let now $\gamma: X^{p} \rightarrow x$ be in $X^{p} / \mathcal{C}$. The quotient of the induced map $\operatorname{ker}(\gamma) \rightarrow X$ is an ind-object and thus defines a lattice of $X$. The functor $\phi$ is essentially surjective and hence an equivalence. 
We also let $\operatorname{Latt}_{\mathcal{C}} / X$ denote the category

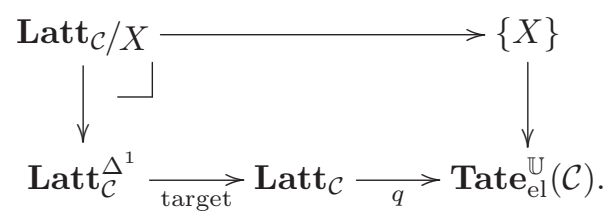

We have a natural fully faithful functor $f_{X}: \operatorname{Latt}_{\mathcal{C}}(X) \rightarrow \operatorname{Latt}_{\mathcal{C}} / X$. We define dually the category $X / \mathbf{L a t t}_{\mathcal{C}}$ and the fully faithful functor $g_{X}: \operatorname{Latt}_{\mathcal{C}}(X) \rightarrow X / \mathbf{L a t t}_{\mathcal{C}}$.

Let $i: \operatorname{Pro}^{\mathbb{U}}(\mathcal{C}) \rightarrow$ Ind $^{\mathbb{V}}$ Pro $^{\mathbb{U}}(\mathcal{C})$ and $j: \operatorname{Ind}^{\mathbb{U}}(\mathcal{C}) \rightarrow$ Pro $^{\mathbb{V}}$ Ind $^{\mathbb{U}}(\mathcal{C})$ denote the canonical embeddings. Let us denote by $\pi_{1}^{p}$ the left Kan extension of $i \pi^{p}$ along the functor $q: \operatorname{Latt}_{\mathcal{C}} \rightarrow \mathbf{T a t e}_{\mathrm{el}}^{\mathbb{U}}(\mathcal{C})$. Dually, we define $\pi_{i}^{!}$the right Kan extension of $j \pi_{i}$ along $q$. We get a diagram

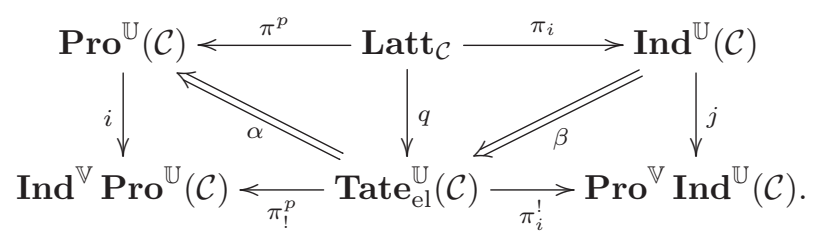

Lemma 3.13. The functor $\pi_{!}^{p}$ is equivalent to the embedding $\mathbf{T a t e}_{\mathrm{el}}^{\mathbb{U}}(\mathcal{C}) \rightarrow$ Ind $^{\mathbb{V}}$ Pro $^{\mathbb{U}}(\mathcal{C})$ defined in Theorem 2.8. The functor $\pi_{i}^{!}$is equivalent to the canonical embedding Tate $_{\mathrm{el}}^{\mathbb{U}}(\mathcal{C}) \rightarrow$ Pro $^{\mathbb{V}} \mathbf{I n d}^{\mathbb{U}}(\mathcal{C})$ defined in Theorem 2.1.

Proof. The statement about $\pi_{i}^{!}$is dual to that about $\pi_{!}^{p}$. Let us prove the latter. Let $s$ denote the section of $\pi^{p}$ mapping a pro-object $X$ to the exact sequence $X \rightarrow X \rightarrow 0$ and let $t$ denote the section of $\pi_{i}$ mapping an ind-object $Y$ to $0 \rightarrow Y \rightarrow Y$. It suffices to prove that the induced functors

$$
\begin{aligned}
& \operatorname{Pro}^{\mathbb{U}}(\mathcal{C}) \stackrel{s}{\rightarrow} \operatorname{Latt}_{\mathcal{C}} \stackrel{q}{\rightarrow} \text { Tate }_{\mathrm{el}}^{\mathbb{U}} \stackrel{\pi^{p}}{\rightarrow} \text { Ind }^{\mathbb{V}} \text { Pro }^{\mathbb{U}}(\mathcal{C}), \\
& \text { Ind }^{\mathbb{U}}(\mathcal{C}) \stackrel{t}{\rightarrow} \operatorname{Latt}_{\mathcal{C}} \stackrel{q}{\rightarrow} \text { Tate }_{\mathrm{el}}^{\mathbb{U}} \stackrel{\pi_{p}^{p}}{\rightarrow} \operatorname{Ind}^{\mathbb{V}} \operatorname{Pro}^{\mathbb{U}}(\mathcal{C})
\end{aligned}
$$

are equivalent to the natural embeddings. Let us first deal with the case of $\operatorname{Pro}^{\mathbb{U}}(\mathcal{C})$. Let $X$ be a pro-object. The image $s(X)$ is a final object in the category $\operatorname{Latt}_{\mathcal{C}} / X$. Hence the canonical map

$$
\alpha_{s(X)}: \pi_{!}^{p} q s(X) \simeq \operatorname{colim}_{Z \bullet \in \operatorname{Latt}_{\mathcal{C}} / X} i \pi^{p}\left(Z^{\bullet}\right) \rightarrow i \pi^{p} s(X) \simeq i(X)
$$

is an equivalence. Let now $Y$ be an ind-object. Let us prove that the category $\mathcal{C} / Y$ of exact sequences $y \rightarrow Y \rightarrow Y / y$ where $y \in \mathcal{C}$ is cofinal in $\operatorname{Latt}_{\mathcal{C}} / Y$. This implies the result, since $Y \simeq \operatorname{colim}_{y \in \mathcal{C} / Y} i(y)$ and $\pi_{!}^{p} q t(Y) \simeq \operatorname{colim}_{Y} \bullet \in \operatorname{Latt}_{\mathcal{C} / Y} i \pi^{p}\left(Y^{\bullet}\right)$.

To prove this cofinality, we will use Quillen's theorem A. Let us denote by $g$ the functor $\mathcal{C} / Y \rightarrow \operatorname{Latt}_{\mathcal{C}} / Y$. Let $Z^{\bullet}=Z^{p} \rightarrow Z \rightarrow Z^{i}$ be a lattice with a map $Z \rightarrow Y$. From Quillen's theorem A (see [HTT, 4.1.3.1]), it suffices to prove that the simplicial 
set

$$
K=Z^{\bullet} /(\mathcal{C} / Y)=\mathcal{C} / Y \underset{\text { Latt }_{\mathcal{C}} / Y}{\times} Z^{\bullet} /\left(\operatorname{Latt}_{\mathcal{C}} / Y\right)
$$

is contractible. We have a diagram

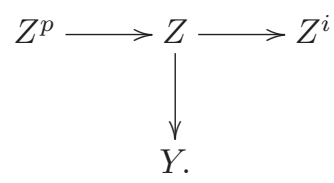

The composite map $Z^{p} \rightarrow Y$, from a pro-object to an ind-object, factors through an object $y \in \mathcal{C}$. Hence we get a commutative diagram

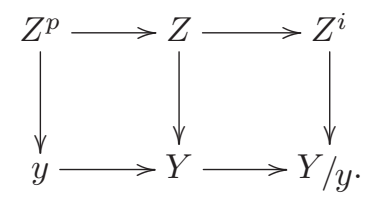

This proves that $K$ is not empty. The category $\mathcal{C}$ admits finite colimits and it follows that $K$ is filtered (as any finite diagram admits an upper bound, namely its colimit). The result is then deduced from [HTT, 5.5.8.7].

Remark 3.14. Let $X$ be a Tate object in $\mathcal{C}$ and let $X_{0}^{\bullet}=\left(X_{0}^{p} \rightarrow X \rightarrow X_{0}^{i}\right)$ and $X_{1}^{\bullet}=$ $\left(X_{1}^{p} \rightarrow X \rightarrow X_{1}^{i}\right)$ be two lattices for $X$. There is a lattice $X^{\bullet}$ for $X$ with maps $X_{0}^{\bullet} \leftarrow X^{\bullet} \rightarrow X_{1}^{\bullet}$. To prove this statement, let us use Theorem 3.6. It defines two Tate diagrams

$$
\begin{aligned}
& \bar{X}_{0}: K^{\text {op }} \rightarrow \operatorname{Ind}^{\mathbb{U}}(\mathcal{C}), \\
& \bar{X}_{1}: L^{\text {op }} \rightarrow \operatorname{Ind}^{\mathbb{U}}(\mathcal{C}),
\end{aligned}
$$

for $X$. Strictifying the identity of $X$ using Theorem 1.2, we get a diagram

$$
\theta: J^{\mathrm{op}} \times \Delta^{1} \rightarrow \operatorname{Ind}^{\mathbb{U}}(\mathcal{C}),
$$

with cofinal maps $\alpha: J \rightarrow K$ and $\beta: J \rightarrow L$ such that $\theta(-, 0) \simeq \bar{X}_{0}(\alpha-)$ and $\theta(-, 1) \simeq$ $\bar{X}_{1}(\beta-)$. The diagram $\theta(-, 1)$ is again a Tate diagram for $X$ and hence defines a lattice $X^{\bullet}$ for $X$. It naturally comes with morphisms $X_{0}^{\bullet} \leftarrow X^{\bullet} \rightarrow X_{1}^{\bullet}$.

We will improve the above remark into the following $\infty$-categorical incarnation of a phenomenon first discovered in [BGW, theorem 6.7].

Theorem 3.15. Let $\mathcal{C}$ be a $\mathbb{U}$-small stable and idempotent complete $(\infty, 1)$-category. For any elementary Tate object $X$ in $\mathcal{C}$, the category $\operatorname{Latt}_{\mathcal{C}}(X)$ is $\mathbb{U}$-small and both filtered and cofiltered. Moreover, the functor $f_{X}: \operatorname{Latt}_{\mathcal{C}}(X) \rightarrow \mathbf{L a t t}_{\mathcal{C}} / X$ is cofinal and the functor $g_{X}: \operatorname{Latt}_{\mathcal{C}}(X) \rightarrow X /$ Latt $_{\mathcal{C}}$ is coinitial.

This theorem, together with Theorem 3.13, implies the following 
Corollary 3.16. Any elementary Tate object $X$ is the colimit in $\operatorname{Ind}^{\mathbb{U}} \operatorname{Pro}^{\mathbb{U}}(\mathcal{C})$

$$
X \simeq \operatorname{colim} X^{p}
$$

and the limit in Pro $^{\mathbb{U}} \operatorname{Ind}^{\mathbb{U}}(\mathcal{C})$

$$
X \simeq \lim X^{i}
$$

where the limit and the colimit are indexed by $\left(X^{p} \rightarrow X \rightarrow X^{i}\right) \in \operatorname{Latt}_{\mathcal{C}}(X)$.

Lemma 3.17. Let $K$ be $a \mathbb{V}$-small simplicial set. Assume that $K$ is filtered and that for any vertex $k \in K$, the simplicial sets $k / K$ and $K / k$ are $\mathbb{U}$-small. Then the simplicial set $K$ is $\mathbb{U}$-small.

Proof. Let $k \in K$ be any vertex. As $K$ is filtered we have

$$
K=\bigcup_{l \in k / K} K / l .
$$

The result follows.

Proof of Theorem 3.15. Let us delay the size issue. We will prove that $\operatorname{Latt}_{\mathcal{C}}(X)$ is filtered and that $f_{X}$ is cofinal. What remains is deduced using the equivalence Tate $^{\mathbb{U}}\left(\mathcal{C}^{\text {op }}\right) \simeq\left(\text { Tate }^{\mathbb{U}}(\mathcal{C})\right)^{\text {op }}$.

We first say that $\operatorname{Latt}_{\mathcal{C}}(X)$ is not empty - see Theorem 3.3. Let now $\bar{X}^{\bullet}: K \rightarrow$ $\operatorname{Latt}_{\mathcal{C}}(X)$ be a finite diagram. We consider the composite diagram

$$
f_{X} \bar{X}^{\bullet}: K \rightarrow \operatorname{Latt}_{\mathcal{C}} / X
$$

The category $\operatorname{Latt}_{\mathcal{C}} / X$ admits finite colimits, and we can hence extend $f_{X} \bar{X}^{\bullet}$ into a colimit diagram $K^{\triangleright} \rightarrow \operatorname{Latt}_{\mathcal{C}} / X$. Let us denote by $Y^{\bullet}=\left(Y^{p} \rightarrow Y \rightarrow Y^{i}\right)$ the colimit. It comes with a map $\psi: Y \rightarrow X$. We get a lattice $X^{\bullet}=\left(X^{p} \rightarrow X \rightarrow X^{i}\right)$ from

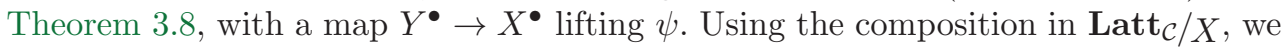
get a diagram $K^{\triangleright} \rightarrow \boldsymbol{L a t t}_{\mathcal{C}} / X$ whose vertices lie in the essential image of $f_{X}$. Since $f_{X}$ is fully faithful, we get a diagram

$$
K^{\triangleright} \rightarrow \operatorname{Latt}_{\mathcal{C}}(X)
$$

extending $\bar{X}^{\bullet}$. This proves the category $\operatorname{Latt}_{\mathcal{C}}(X)$ is filtered.

We now have to prove the functor $f_{X}$ is cofinal. Let $Y^{\bullet} \in \operatorname{Latt}_{\mathcal{C}} / X$. From Theorem 3.8, we deduce that the category

$$
\mathcal{D}=\operatorname{Latt}_{\mathcal{C}}(X) \underset{\operatorname{Latt}_{\mathcal{C} / X}}{\times} Y^{\bullet} /\left(\operatorname{Latt}_{\mathcal{C}} / X\right)
$$

is not empty. Take a finite diagram $K \rightarrow D$. We see using the same argument as above that it extends into a diagram $K^{\triangleright} \rightarrow \mathcal{D}$. In particular, $\mathcal{D}$ is filtered and hence the underlying simplicial set is contractible. We conclude using Quillen's theorem A - see [HTT, 4.1.3.1].

To see that $\operatorname{Latt}_{\mathcal{C}}(X)$ is essentially $\mathbb{U}$-small, we now use Theorem 3.17 and Theorem 3.12. 


\section{K-theory}

In this section, we will prove Theorem 1. The strategy of the proof is inspired by that in the case of exact categories, which can be found in [Sai].

Definition 4.1. Let $\mathcal{C} \rightarrow \mathcal{D}$ be a fully faithful exact functor between $\mathbb{V}$-small stable and idempotent complete $(\infty, 1)$-categories. We denote by $\mathcal{D} / \mathcal{C}$ the cofibre of the functor $\mathcal{C} \rightarrow \mathcal{D}$ in the category of ( $\mathbb{V}$-small) stable and idempotent complete $(\infty, 1)$ categories. Note that its existence is guarantied by [BGT, Part 5]. Let us call $\mathcal{D} / \mathcal{C}$ the Verdier quotient of $\mathcal{D}$ by $\mathcal{C}$.

Proposition 4.2. Let $\mathcal{C}$ be $a \mathbb{V}$-small stable and idempotent complete $(\infty, 1)$-category. The commutative diagram

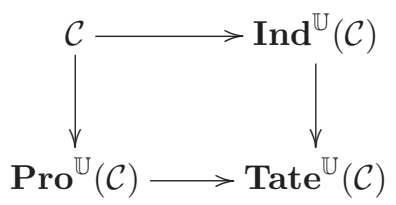

induces an equivalence between the Verdier quotients

$$
\operatorname{Ind}^{\mathbb{U}}(\mathcal{C}) / \mathcal{C} \stackrel{\sim}{\rightarrow} \operatorname{Tate}^{\mathbb{U}}(\mathcal{C}) / \text { Pro }^{\mathbb{U}}(\mathcal{C})
$$

Proof. Let us fix the following notation

$$
\begin{array}{ll}
I^{\mathbb{V}} \mathcal{C}=\operatorname{Ind}^{\mathbb{V}}(\mathcal{C}), & I^{\mathbb{V}} \mathrm{IC}=\operatorname{Ind}^{\mathbb{V}} \operatorname{Ind}^{\mathbb{U}}(\mathcal{C}), \\
\mathrm{I}^{\mathbb{V}} \mathrm{PC}=\operatorname{Ind}^{\mathbb{V}} \text { Pro }^{\mathbb{U}}(\mathcal{C}), & \mathrm{I}^{\mathbb{V}} \mathrm{T} \mathcal{C}=\operatorname{Ind}^{\mathbb{V}} \text { Tate }^{\mathbb{U}}(\mathcal{C}) .
\end{array}
$$

We also set

$$
\mathcal{E}=\operatorname{Ind}^{\mathbb{V}}\left(\operatorname{Ind}^{\mathbb{U}}(\mathcal{C}) / \mathcal{C}\right) \quad \text { and } \quad \mathcal{E}^{\prime}=\operatorname{Ind}^{\mathbb{V}}\left(\operatorname{Tate}^{\mathbb{U}}(\mathcal{C}) / \text { Pro }^{\mathbb{U}}(\mathcal{C})\right) .
$$

The commutative diagram

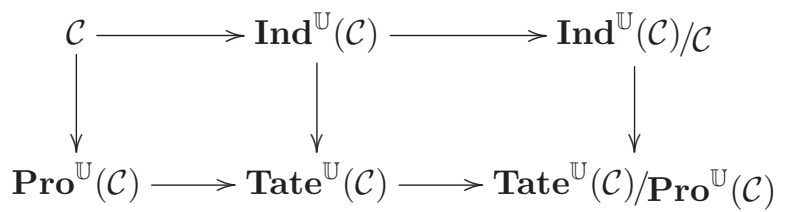

induces the diagram of adjunctions between presentable stable $(\infty, 1)$-categories

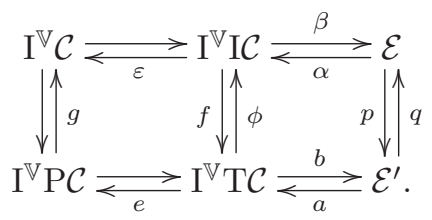

We have represented here the left adjoints on top or on the left of their right adjoint. It follows from [BGT, 5.12 and 5.13] that the two lines in the above diagram are cofibre sequences of presentable stable $(\infty, 1)$-categories. Since $\operatorname{Ind}^{\mathbb{U}}(\mathcal{C}) / \mathcal{C}($ resp. 
$\left.\operatorname{Tate}^{\mathbb{U}}(\mathcal{C}) / \operatorname{Pro}^{\mathbb{U}}(\mathcal{C})\right)$ is idempotent complete, it is equivalent to the category of compact objects in $\mathcal{E}$ (resp. $\mathcal{E}^{\prime}$ ). It hence suffices to prove that $p$ and $q$ are equivalences. We will prove the sufficient assertions:

(a) The functor $p$ is fully faithful.

(b) The functor $q$ is conservative.

Let us start with assertion (a). Using [BGT, 5.5], we deduce that both $a$ and $\alpha$ are fully faithful. Moreover, the functor $f$ is also fully faithful, and it thus suffices to prove the equivalence $f \alpha \simeq a p$. We have $b f \alpha \simeq p \beta \alpha \simeq p$. It is now enough to prove that $f \alpha$ has values in the essential image of $a$ (so that $a b f \alpha \simeq f \alpha$ ). To do so, we will show that for any object $x \in \mathrm{I}^{\mathbb{V}} \mathrm{IC}$, if $\varepsilon(x)$ vanishes, then so does ef $(x)$. Let $\bar{x}: K \rightarrow \operatorname{Ind}^{\mathbb{U}}(\mathcal{C})$ denote a $\mathbb{V}$-small filtered diagram whose colimit in $\mathrm{I}^{\mathbb{V}} \mathrm{IC}$ is $x$. Let also $\bar{y}: L^{\mathrm{op}} \rightarrow \mathcal{C}$ be a $\mathbb{U}$-small cofiltered diagram. We denote by $y$ its limit in $\operatorname{Pro}^{\mathbb{U}}(\mathcal{C})$. The functor $e$ preserves filtered colimits (as its left adjoint preserves compact objects). The image ef $f(x)$ is therefore the functor Pro $\mathbb{U}^{\mathbb{U}}(\mathcal{C}) \rightarrow$ sSets mapping $y$ to the simplicial set

$$
\underset{k \in K}{\operatorname{colim}} \operatorname{colim}_{l \in L} \operatorname{Map}_{\mathbf{I n d}^{\mathbb{U}}(\mathcal{C})}(\bar{y}(l), \bar{x}(k)) \simeq \operatorname{colim}_{l \in L} \operatorname{colim}_{k \in K} \operatorname{Map}_{\mathbf{I n d}^{\mathbb{U}}(\mathcal{C})}(\bar{y}(l), \bar{x}(k)) .
$$

On the other hand, the assumption $\varepsilon(x)=0$ implies that for any $c \in \mathcal{C}$, the space

$$
\underset{k \in K}{\operatorname{colim}} \operatorname{Map}_{\mathbf{I n d}^{\mathbb{U}}(\mathcal{C})}(c, \bar{x}(k))
$$

is contractible. It follows from [HTT, 5.5.8.7] that ef $(x)$ vanishes.

We can now focus on assertion (b). Since $q$ preserves exact sequences and $a$ is fully faithful, it suffices to prove that if $z \in \mathrm{I}^{\mathbb{V}} \mathrm{TC}$ is such that both $\phi(z)$ and $e(z)$ vanish, then so does $z$. We can see $z$ as a functor Tate ${ }^{\mathbb{U}}(\mathcal{C})^{\text {op }} \rightarrow$ sSets preserving finite limits while $\phi(z)$ and $e(z)$ are its restriction respectively to $\operatorname{Ind}^{\mathbb{U}}(\mathcal{C})^{\text {op }}$ and $\operatorname{Pro}^{\mathbb{U}}(\mathcal{C})$. As Tate ${ }^{\mathbb{U}}(\mathcal{C})$ is generated by ind- and pro-objects under finite limits and retracts, we deduce that $z$ is equivalent to 0 .

Corollary 4.3. Let $\mathcal{C}$ be $a \mathbb{V}$-small stable and idempotent complete $(\infty, 1)$-category. The spectrum of non-connective $K$-theory of $\operatorname{Tate}^{\mathbb{U}}(\mathcal{C})$ is the suspension of the nonconnective $K$-theory of $\mathcal{C}$ :

$$
\mathbb{K}\left(\operatorname{Tate}^{\mathbb{U}}(\mathcal{C})\right) \simeq \Sigma \mathbb{K}(\mathcal{C})
$$

Remark 4.4. This corollary is an $\infty$-categorical version of a theorem of Sho Saito in exact 1-categories in [Sai].

Proof. Let us use the notation $\mathrm{IC}=\operatorname{Ind}^{\mathbb{U}}(\mathcal{C}), \mathrm{PC}=\operatorname{Pro}^{\mathbb{U}}(\mathcal{C})$ and $\operatorname{TC}^{-}=\operatorname{Tate}^{\mathbb{U}}(\mathcal{C})$. Because the K-theory functor preserves cofibre sequences of stable categories (see [BGT, sect. 9]), we get two exact sequences in the $(\infty, 1)$-category of spectra

$$
\begin{gathered}
\mathbb{K}(\mathcal{C}) \rightarrow \mathbb{K}(\mathrm{IC}) \rightarrow \mathbb{K}\left(\text { Ind }^{\mathbb{U}}(\mathcal{C}) / \mathcal{C}\right), \\
\mathbb{K}(\mathrm{PC}) \rightarrow \mathbb{K}(\mathrm{TC}) \rightarrow \mathbb{K}\left(\operatorname{Ind}^{\mathbb{U}}(\mathcal{C}) / \mathcal{C}\right) .
\end{gathered}
$$

The vanishing of $\mathbb{K}(\mathrm{PC})$ and $\mathbb{K}(\mathrm{IC})$ - since those categories contain countable sums concludes the proof. 


\section{An application: families of Tate complexes}

In this last section, we will study Tate complexes in (derived) algebraic geometry. In this context, one should think of Tate complexes as structured infinite dimensional vector bundles (or more generally quasicoherent complexes). In this section, we will produce additive invariants on such Tate complexes, out of the common additive invariants of finite dimensional vector bundles (or perfect complexes), using our Theorem 4.3.

As an example of such additive invariants, we will be able to define the dimension of a Tate complex (or rather its Euler characteristic). Given a finite dimensional vector bundle on a variety $X$, the dimension can be seen as a locally constant function $X \rightarrow \mathbb{Z}$ - or equivalently as a class in $\mathrm{H}^{0}(X, \mathbb{Z})$. The shift in $\mathrm{K}$-theory we proved in Theorem 4.3 will allow us to define the dimension of a Tate complex as a class in $\mathrm{H}^{1}(X, \mathbb{Z})$ - or equivalently as a $\mathbb{Z}$-torsor over $X$.

Another very interesting example of such an invariant will be the determinant of a Tate complex. This determinant will be a class in $\mathrm{H}^{2}\left(X, \mathbb{G}_{m}\right)$, hence classifying a gerbe with lien $\mathbb{G}_{m}$ over $X$.

Note that this question was, at least partially, addressed in [Dri] or [OZ, section 3.2] in the context of (usual) algebraic geometry.

In the work, we will focus on the derived algebro-geometric setting, that has not been covered in any previous work.

We start with a short introduction to derived algebraic geometry.

DAG IN A NUTShell Let us assume $k$ is a field. First introduced by Toën and Vezzosi in [HAG2], derived algebraic geometry is a generalisation of algebraic geometry in which we replace commutative algebras over $k$ by simplicial commutative algebras up to homotopy. We refer to [Toë] for a recent survey of this theory.

Derived algebraic geometry allows us to study ill-behaved geometric situations. The most emblematic example is the study of non-generic intersections, or of quotients by a wild action. Note that usual objects of algebraic geometry - varieties, schemes, algebraic spaces or stacks - embed in derived algebraic geometry. Another nice feature of this theory is the cotangent bundle. If we usually require smoothness to define a tangent bundle, dual to the cotangent, it is no longer needed in derived algebraic geometry (we only need finiteness conditions). The main trick is to consider the (co)tangent bundle not as a quasi-coherent sheaf, but as a complex of such. The category of quasi-coherent complexes becomes a central object in this context. This category is actually a stable and idempotent complete $(\infty, 1)$-category. This core example of such a category motivates the results of this article. The derived category of quasi-coherent complex of a derived stack $X$ admits a full-subcategory $\operatorname{Perf}(X)$ of socalled perfect complexes. Perfect complexes are to complexes what finitely generated projective modules are to modules. In particular, they behave regarding duality.

We will denote by $\mathbf{s C A l g}$ the $(\infty, 1)$-category of simplicial commutative algebras over $k$. It is the $(\infty, 1)$-localization of a model category along weak equivalences. Let us denote $\mathbf{d A f f} \mathbf{A f}_{k}$ the opposite $(\infty, 1)$-category of $\mathbf{s} \mathbf{C A l g}{ }_{k}$. It is the category of derived affine schemes over $k$.

A derived prestack is a presheaf $\mathbf{d A f f}{ }_{k}^{\text {op }} \simeq \mathbf{s} \mathbf{C A} \lg _{k} \rightarrow \mathbf{s S e t s}$. We will thus write $\mathcal{P}\left(\mathbf{d A f f}_{k}\right)$ for the $(\infty, 1)$-category of derived prestacks. A derived stack is a prestack 
satisfying a descent condition. For the purpose of this paper, we will only consider the Nisnevich topology. We will denote by $\mathbf{d} \mathbf{S t} t_{k}^{\text {Nis }}$ the $(\infty, 1)$-category of derived Nisnevich stacks. It comes with an adjunction

$$
(-)^{+}: \mathcal{P}\left(\mathbf{d A f f}_{k}\right) \rightleftarrows \mathbf{d S t}_{k}^{\mathrm{Nis}},
$$

where the left adjoint $(-)^{+}$is called the stackification functor.

Let Perf denote the derived stack of perfect complexes $A \mapsto \operatorname{Perf}(A)$. Let also $\mathrm{K}$ denote the connective K-theory functor (seen as a group object in spaces).

Definition 5.1. Let us define the groups in prestacks

$$
\begin{aligned}
& \mathrm{K}^{\text {Perf }}: A \mapsto \mathrm{K}(\operatorname{Perf}(A)), \\
& \mathrm{K}^{\text {Tate }}: A \mapsto \mathrm{K}\left(\operatorname{Tate}^{\mathbb{U}}(\operatorname{Perf}(A))\right) .
\end{aligned}
$$

We also define the prestack of Tate complexes

$$
\text { Tate: } A \mapsto \operatorname{Tate}^{\mathbb{U}}(\operatorname{Perf}(A)) \text {. }
$$

From Theorem 4.3, we get a fibre sequence of prestacks

$$
\mathrm{BK}^{\text {Perf }} \longrightarrow \mathrm{K}^{\text {Tate }} \longrightarrow \mathrm{K}_{0}^{\text {Tate }} \text {. }
$$

Lemma 5.2. The prestack $\mathrm{K}_{0}^{\text {Tate }}$ vanishes Nisnevich-locally. It follows that the map $\mathrm{BK}^{\text {Perf }} \rightarrow \mathrm{K}^{\text {Tate }}$ is a Nisnevich-local equivalence.

Proof. Is suffices to prove that for any Henselian simplicial commutative algebra $A$, we have

$$
\mathrm{K}_{-1}(\operatorname{Perf}(A)) \simeq \mathrm{K}_{0}\left(\operatorname{Tate}^{\mathbb{U}}(\operatorname{Perf}(A))\right) \simeq 0 .
$$

Recall that $A$ is Henselian if and only if $\pi_{0}(A)$ is. Using the Bass exact sequences, we get

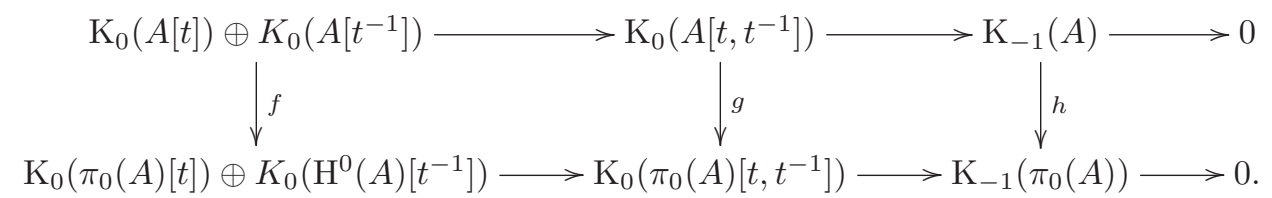

Since $\mathrm{K}_{0}$ only depends on the non-derived part of an affine scheme (see [Wal, 2.3.2]), both $f$ and $g$ are isomorphisms and hence so is $h$. We can thus restrict to the nonderived case - which can be found in [Dri, theorem 3.7].

Theorem 5.3. Let $i$ be any additive invariant of perfect complexes: it can then be encoded as a group morphism $i: \mathrm{K}^{\text {Perf }} \rightarrow G$ for any group object $G$.

The invariant $i$ induces an additive invariant $[i]$ of Tate complexes:

$$
[i]: \mathrm{K}^{\text {Tate }} \rightarrow \mathrm{B} G,
$$

with values in the classifying stack $\mathrm{B} G$. In particular, for any derived algebraic stack $X$ and any Tate complex $E$ over $X$, we get a $G$-bundle classified by the map

$$
X \stackrel{E}{\longrightarrow} \text { Tate } \longrightarrow \mathrm{K}^{\text {Tate }} \stackrel{[i]}{\longrightarrow} \mathrm{B} G \text {. }
$$


Remark 5.4. In the theorem above, we only need the map $i$ to preserve the group structure. It does not need to preserve the commutativity constraints.

Proof. From Theorem 5.2, we see that the stack $\left(\mathrm{K}^{\text {Tate }}\right)^{+}$associated to the prestack $\mathrm{K}^{\text {Tate }}$ is equivalent to that associated to $\mathrm{BK}^{\text {Perf }}$. We can hence form $\mathrm{B} i$ : $\mathrm{BK}^{\text {Perf }} \rightarrow$ $\mathrm{B} G$. Let us denote by $\delta:\left(\mathrm{B} \mathrm{K}^{\text {Perf }}\right)^{+} \rightarrow \mathrm{B} G$ the map of stacks obtained by stackifying B $i$. We can hence set

$$
[i]: \mathrm{K}^{\text {Tate }} \longrightarrow\left(K^{\text {Tate }}\right)^{+} \simeq\left(\mathrm{B} \mathrm{K}^{\text {Perf }}\right)^{+} \stackrel{\delta}{\longrightarrow} \mathrm{B} G .
$$

A motivating Example We define the determinantal anomaly to be the invariant [Det] associated to the determinant Det: $\mathrm{K}^{\text {Perf }} \rightarrow \mathrm{B} \mathbb{G}_{m}$ :

$$
\text { [Det]: } \mathrm{K}^{\text {Tate }} \rightarrow \mathrm{B} \mathrm{B} \mathbb{G}_{m} \simeq \mathrm{K}\left(\mathbb{G}_{m}, 2\right) .
$$

In particular, any Tate object $E$ over a derived stack $X$ defines a determinantal anomaly $\left[\operatorname{Det}_{E}\right] \in \mathrm{H}_{\mathrm{Nis}}^{2}\left(X, \mathcal{O}_{X}^{\times}\right)$. Similarly, applying Theorem 5.3 to the Euler characteristic $i=\operatorname{Dim}=\chi: \mathrm{K}^{\text {Perf }} \rightarrow \mathbb{Z}$, we get the dimensional torsor $\left[\operatorname{Dim}_{E}\right]=\left[\chi_{E}\right] \in$ $\mathrm{H}_{\mathrm{Nis}}^{1}(X, \mathbb{Z})$ (see $[$ Dri $]$ ).

The above construction is for instance useful in the following application. In [Hen], the author introduces the $d$-dimensional formal loops space $\mathcal{L}^{d}(X)$ with values in a nice enough derived stack. It is a derived stack representing maps from the punctured formal neighbourhood $\operatorname{Spec}\left(k \llbracket t_{1}, \ldots, t_{d} \rrbracket\right) \backslash\{0\}$ to $X$. We prove in loc. cit. that the tangent of this formal loops space is a Tate object over $\mathcal{L}^{d}(X)$. It follows from the above construction the existence of a class, called the determinantal class

$$
\left[\operatorname{det}_{\mathbb{T}}\right] \in \mathrm{H}_{\mathrm{Nis}}^{2}\left(\mathcal{L}^{d}(X), \mathcal{O}^{\times}\right) .
$$

This class generalises the class introduced by Kapranov and Vasserot in [KV1], that is proved to be an obstruction to the existence of sheaves of chiral differential operators.

Note that the determinantal gerbe is in general not trivial. The case where $X$ is the stack B $G$ classifying $G$-bundles, for an algebraic group $G$, this determinantal class determines a non-trivial central extension of the shifted tangent dg-Lie algebra of $\mathcal{L}^{d}(X)$ at the neutral element. This central extension is studied in [FHK], as it provides a higher dimensional analogue to the Kac-Moody extensions. Indeed, when $d=1$, we find back $\mathfrak{g} \otimes k((t))$ and its usual extension by a central charge.

\section{References}

[BGT] Andrew J. Blumberg, David Gepner and Gonçalo Tabuada: A universal characterization of higher algebraic K-theory. Geom. Topol. 17 (2013), no. $2,733-838$.

[BGW] Oliver Bräunling, Michael Gröchenig and Jesse Wolfson: Tate objects in exact categories. Mosc. Math. J. 16 (2016), 433-504.

[Dri] Vladimir DRINFELD: Infinite-dimensional vector bundles in algebraic geometry (An introduction). 2004, available at http://arxiv.org/abs/ math/0309155. 
[FHK] Giovanni Faonte, Benjamin Hennion and Mikhail Kapranov: Higher Kac-Moody algebras and derived moduli spaces of G-bundles. 2017, available at https://arxiv.org/abs/1701.01368.

[HAG2] Bertrand Tö̈N and Gabriele Vezzosi: Homotopical algebraic geometry II: geometric stacks and applications. Mem. Amer. Math. Soc. 193 (2008), no. 902, pp. 257-372.

[HAlg] Jacob LuRIE: Higher algebra. February 15, 2012, available at http: //www.math.harvard.edu/ lurie/papers/HigherAlgebra.pdf.

[Hen] Benjamin Hennion: Higher dimensional formal loop spaces. Ann. Sci. École Norm. Sup. (4), 50 (2017), no. 3, 609-663.

[HTT] Jacob LURIE: Higher Topos Theory, volume 170 of Annals of Mathematics Studies. Princeton University Press, 2009.

[KV1] Mikhail KAPRANOV and Eric VAssERot: Vertex algebras and the formal loop space. Publ. Math. Inst. Hautes Études Sci. 100 (2004), pp. 209-269.

[KV2] Mikhail Kapranov and Eric VAsserot: Formal loops II: A local Riemann-Roch theorem for determinantal gerbes. Ann. Sci. École Norm. Sup. (4) 40 (2007), no. 1, pp. 113-133.

[OZ] Denis Osipov and Xiewen Zhu: A categorical proof of the Parshin reciprocity laws on algebraic surfaces. Algebra Number Theory 5 (2011), no. 3, pp. 289-337.

[Pre] Luigi Previdi: Generalized Tate space. PhD thesis, Yale University, 2010.

[Sai] Sho SAITo: On Previdi's delooping conjecture for K-theory. 2013, available at http://arxiv.org/abs/1203.0831.

[Toë] Bertrand Tö̈n: Derived algebraic geometry. EMS Surv. Math. Sci. 1 (2014), no. 2, 153-240.

[Wal] Friedhelm Waldhausen: Algebraic K-theory of spaces. In Algebraic and Geometric Topology, volume 1126 of Lecture Notes in Math., pp. 318-419. Springer, 1983.

Benjamin Hennion benjamin.hennion@math.u-psud.fr

Max-Planck Institut für Mathematik, Vivatsgasse 7, Bonn, Germany 